\title{
Ready, Set, Verify! Applying hs-to-coq to real-world Haskell code
}

\author{
JOACHIM BREITNER \\ DFINITY Stiftung, Zug, Switzerland \\ (e-mail: joachim@dfinity.org)
}

\author{
ANTAL SPECTOR-ZABUSKY(i) \\ University of Pennsylvania, Philadelphia, USA \\ (e-mail: antals@cis.upenn.edu) \\ YAO LID \\ University of Pennsylvania, Philadelphia, USA \\ (e-mail: liyao@cis.upenn.edu) \\ CHRISTINE RIZKALLAH \\ University of New South Wales, Sydney, Australia \\ (e-mail: c.rizkallah@unsw.edu.au) \\ JOHN WIEGLEY \\ DFINITY Stiftung, Zug, Switzerland \\ (e-mail: john@dfinity.org) \\ JOSHUA COHEN \\ University of Pennsylvania, Philadelphia, USA \\ (e-mail: joscoh@sas. upenn.edu) \\ STEPHANIE WEIRICH \\ University of Pennsylvania, Philadelphia, USA \\ (e-mail: sweirich@cis.upenn.edu)
}

\begin{abstract}
Good tools can bring mechanical verification to programs written in mainstream functional languages. We use hs-to-coq to translate significant portions of Haskell's containers library into Coq, and verify it against specifications that we derive from a variety of sources including type class laws, the library's test suite, and interfaces from Coq's standard library. Our work shows that it is feasible to verify mature, widely used, highly optimized, and unmodified Haskell code. We also learn more about the theory of weight-balanced trees, extend hs-to-coq to handle partiality, and - since we found no bugs - attest to the superb quality of well-tested functional code.
\end{abstract}

\section{Introduction}

What would it take to tempt functional programmers to verify their code?

Certainly, better tools would help. We see that functional programmers who use dependently typed languages or proof assistants, such as Coq (The Coq development team, 2016), Agda (Bove et al., 2009), Idris (Brady, 2017), and Isabelle (Nipkow et al., 2002), 
do verify their code, since their tools allow it. However, adopting these platforms means rewriting everything from scratch. What about the verification of existing code, such as libraries written in mature languages like Haskell?

Haskell programmers can reach for LiquidHaskell (Vazou et al., 2014) which smoothly integrates the expressive power of refinement types with Haskell, using SMT solvers for fully automatic verification. But some verification endeavors require the full strength of a mature interactive proof assistant like Coq. The hs-to-coq tool, developed by SpectorZabusky et al. (2018), translates Haskell types, functions, and type classes into equivalent Coq code - a form of shallow embedding - which can be verified just like normal Coq definitions.

But can this approach be used for more than the small, textbook-sized examples it has been applied to so far? Yes, it can! In this work, we use hs-to-coq to translate and verify the two set data structures from Haskell's containers package. ${ }^{1}$ This codebase is not a toy. It is decades old, highly tuned for performance, type-class polymorphic, and implemented in terms of low-level features like bit manipulation operators and raw pointer equality. It is also an integral part of the Haskell ecosystem. We make the following contributions:

- We demonstrate that hs-to-coq is suitable for the verification of unmodified, real-world Haskell libraries. By "real-world", we mean code that is up-to-date, in common use, and optimized for performance. In Section 2, we describe the containers library in more detail and discuss why it fits this description.

- We present a case study not just of verifying a popular Haskell library, but also of developing a good specification of that library. This process is worth consideration because it is not at all obvious what we mean when we say that we have "verified" a library. Section 4 discusses the techniques that we have used to construct a rich, two-sided specification; one that draws from diverse, cross-validated sources and yet is suitable for verification.

- We extend hs-to-coq and its associated standard libraries to support our verification goal. In particular, in Section 5, we describe the challenges that arise when translating the Data. Set and Data. IntSet modules, and our solutions. Notably, we drop the restriction in previous work (Spector-Zabusky et al., 2018) that the input of the translation must be intrinsically total. Instead, we show how to safely defer reasoning about incomplete pattern matching and potential nontermination to later stages of the verification process.

- We increase confidence in the translation done of hs-to-coq. In one direction, properties of the Haskell test suite turn into Coq theorems that we prove. In the other direction, the translated code, when extracted back to Haskell, passes the original test suite.

- We provide new implementation-agnostic insight into the verification of the weightbalanced tree data structure, as we describe in Section 6. In particular, we find the right precondition for the central balancing operations needed to verify the particular variant used in Data. Set.

1 Specifically, we target version 0.5 .11 .0 , which was released on January 22, 2018 and was the most recent release of this library at the time of publication; it is available at https://github.com/haskell/ containers/tree/v0.5.11.0. 
Our work provides a rich specification for Haskell's finite set libraries that is directly and mechanically connected to the current implementation. As a result, Haskell programmers can be assured that these libraries behave as expected. Of course, there is a limit to the assurances that we can provide through this sort of effort. We discuss the verification gap and other limitations of our approach in Section 7.

We would like to have been able to claim the contribution of finding bugs in containers, but there simply were none. Still, our efforts resulted in improvements to the containers library. First, an insight during the verification process led to an optimization that makes the Data. Set.union function $4 \%$ faster. Second, we discovered an incompleteness in the specification of the validity checker used in the test suite.

The tangible artifacts of this work have been incorporated into the hs-to-coq distribution and are available as open source tools and libraries. ${ }^{2}$

\section{The containers library}

We selected the containers library for our verification efforts because it is a critical component of the Haskell ecosystem. With over 4000 publicly available Haskell packages using containers, it is the third-most depended-on package of the Haskell package repository Hackage, after base and bytestring. ${ }^{3}$

The containers library is both mature and highly optimized. It has existed for over a decade and has undergone many significant revisions in order to improve its performance. It contains seven container data structures, covering support for finite sets (Data. Set and Data.IntSet), finite maps (Data.Map and Data.IntMap), sequences (Data.Sequence), graphs (Data.Graph), and trees (Data.Tree). However, most users of the containers library only use the map and set modules ${ }^{4}$ moreover, the map modules are essentially analogues of the set modules. Therefore, we focus on the verification of Data. Set and Data. IntSet in the majority of this work. Furthermore, we used our experience verifying Data. Set to also verify Data.Map.Strict, as we describe in Section 5.9.

\subsection{Weight-balanced trees}

The Data. Set module implements finite sets using weight-balanced binary search trees. The definition of the Set datatype in this module, along with its membership function, is given in Figure $1 .^{5}$

These sets and operations are polymorphic over the element type and require only that this type is linearly ordered, as expressed by the Ord constraint on the member function. The member function descends the ordered search tree to determine whether it contains a particular element.

2 The full repository for hs-to-coq is at https://github.com/antalsz/hs-to-coq; for the version connected to this work, see the JFP-containers tag, which can be accessed at https://github.com/ antalsz/hs-to-coq/tree/JFP-containers. The examples/containers directory contains the verification of containers; consult examples/containers/README.md for a guide to the relevant portions of the code.

3 http://packdeps. haskellers.com/reverse

4 We calculated that $78 \%$ of the packages on Hackage that depend on containers use only sets and maps.

5 All code listings in this paper are manually reformatted and may omit module names from fully qualified names. 


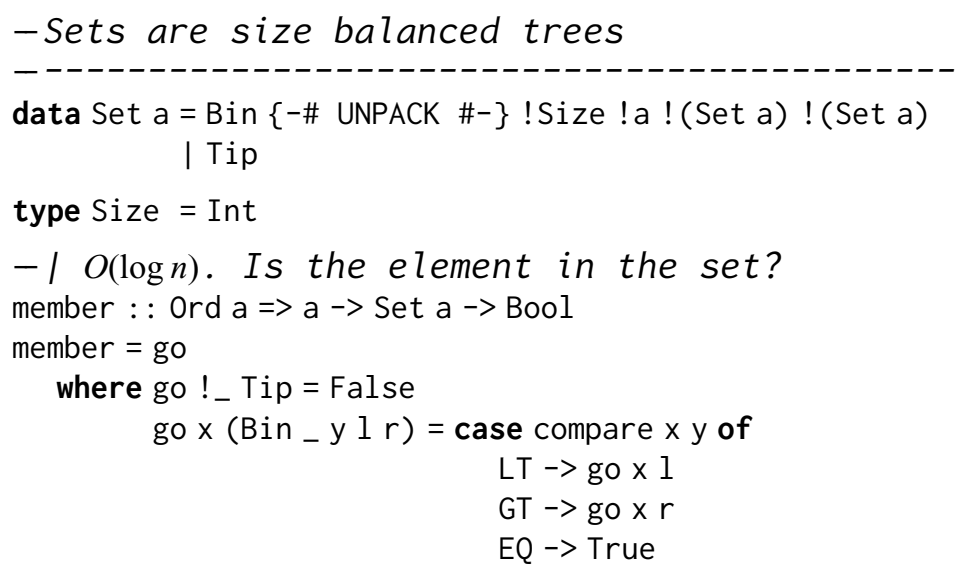

Fig. 1. The Set data type and its membership function, from http://hackage.haskell.org/ package/containers-0.5.11.0/docs/src/Data. Set. Internal.html\#Set.

The Size component stored with the Bin constructor is used by the operations in the library to ensure that the tree stays balanced. The implementation maintains the balancing invariant

$$
s_{1}+s_{2} \leq 1 \vee\left(s_{1} \leq 3 s_{2} \wedge s_{2} \leq 3 s_{1}\right),
$$

where $s_{1}$ and $s_{2}$ are the sizes of the left and right subtrees of a Bin constructor. This definition is based on the description by Adams (1992), who modified the original weightbalanced tree proposed by Nievergelt \& Reingold (1972). Thanks to this balancing, operations such as insertion, membership testing, and deletion take time logarithmic in the size of the tree.

This type definition has been tweaked to improve the performance of the library. The ! annotations indicate that all components should be strictly-evaluated; whenever a value constructed by Bin is evaluated, so are all of its strictly evaluated fields. Because the Size field is strict, it can be unpacked; this is a size and speed optimization that stores the machine word directly with the constructor, rather than storing a pointer to a boxed heap representation thereof.

\subsection{Big-endian Patricia trees}

The Data. IntSet module also provides search trees, but these are specialized to values of type Int to provide more efficient operations, especially union. This implementation is based on big-endian Patricia trees, as proposed in Morrison's work on PATRICIA (1968) and described in a pure functional setting by Okasaki \& Gill (1998).

The definition of this data structure is shown in Figure 2. The core idea is to use the bits of the stored values to decide in which subtree of a node they should be placed. In a node Bin p m s1 s2, the mask $m$ has exactly one bit set. All bits higher than the mask bit are equal in all elements of that node; they form the prefix $p$. The mask bit is the highest bit that is not shared by all elements. In particular, all elements in $s 1$ have this bit cleared, while all elements in 52 have it set. When looking up a value $x$, the mask bit of $x$ tells us into which branch to descend. 


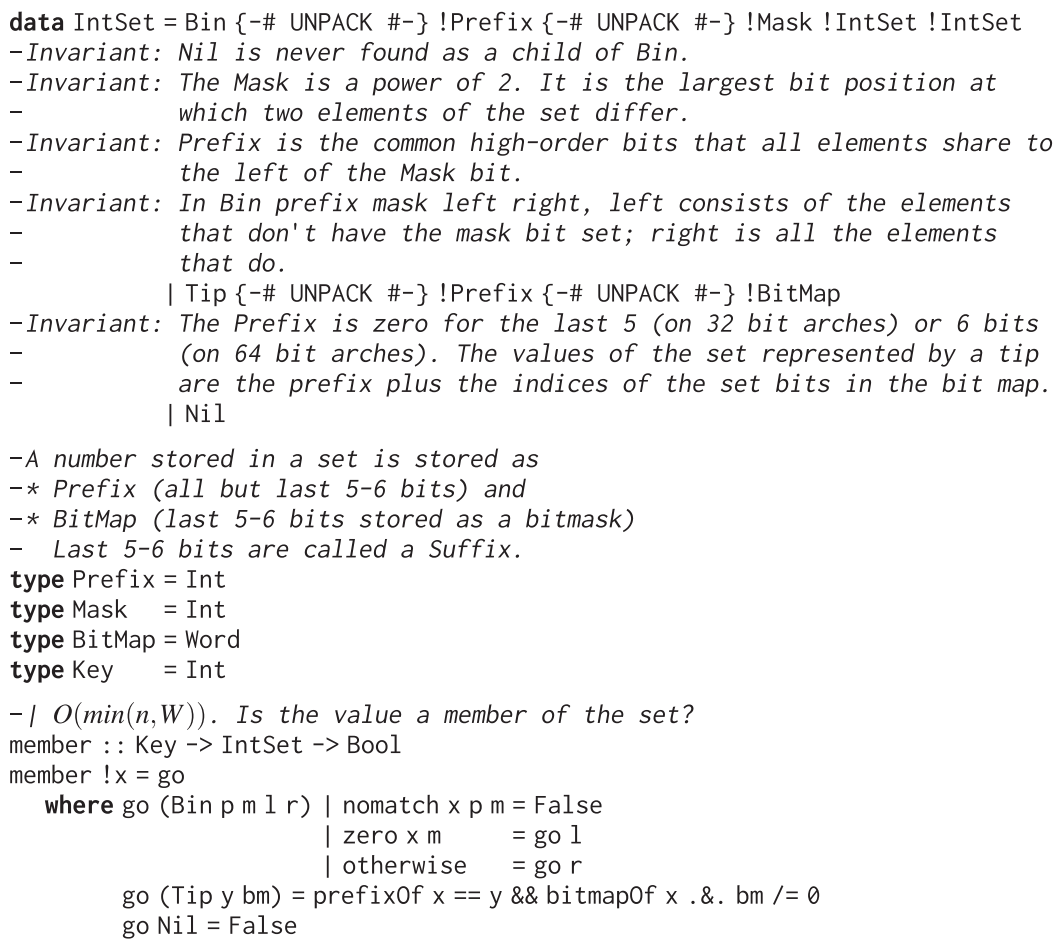

Fig. 2. The IntSet data type and its membership function, from http://hackage.haskell.org/ package/containers-0.5.11.0/docs/src/Data. IntSet. Internal.html\#IntSet.

Instead of storing a single value at the leaf of the tree, the implementation in containers improves time and space performance by storing the membership information of consecutive numbers as the bits of a machine-word-sized bitmap in the Tip constructor. ${ }^{6}$

The Nil constructor is the only way to represent an empty tree, and will never occur as the child of a Bin constructor. Every well-formed IntSet is either made of Bins and Tips, or a single $\mathrm{Nil}$.

\subsection{A history of performance tuning}

The history of the Data. Set module can be traced back to 2004, when a number of competing search tree implementations were debated in the "Tree Wars" thread on the Haskell libraries mailing list. Benchmarks showed that Daan Leijen's implementation had the best performance, and it was added to containers in 2005 as Data. Set. ${ }^{7}$

In 2010, Milan Straka thoroughly evaluated the performance of the containers library and implemented a number of performance tweaks (Straka, 2010). For example:

When balancing a node, the function balance checked the balancing condition and called one of the four rotating functions, which rebuilt the tree using smart constructors. This resulted in a repeated pattern matching, which was unnecessary.

\footnotetext{
6 Although this feature was contributed in 2011 by the first author, he certainly did this without having an eventual formal verification in mind.

7 https://github.com/haskell/containers/commit/bbbba97c
} 
We rewrote the balance function to contain all the logic and to use as few pattern matches as possible. That resulted in significant performance improvements in all Set methods that modify a given set.

This change ${ }^{8}$ replaced a fairly readable balance and several small and descriptive helper functions with a single dense block of code. A later change ${ }^{9}$ by Straka created two copies of this dense, complicated balance function, each specialized and optimized for different preconditions.

Adams (1992) describes two algorithms for union, intersection, and difference: "hedge union" and "divide and conquer". Originally containers used the former, but in 2016 its maintainers switched to the latter, ${ }^{10}$ again based on performance measurement.

The module Data. IntSet (and Data. IntMap) has been around even longer. Okasaki \& Gill mention in their 1998 paper (Okasaki \& Gill, 1998) that GHC had already made use of IntSet and IntMap for several years. In 2011, the Data. IntSet module was rewritten to use machine words as bit maps in the leaves of the tree, as discussed at the end of Section 2.2. ${ }^{11}$ This moved the containers library further away from the literature on Patricia trees and introduced a fair amount of low-level bit-twiddling operations (e.g., highestBitMask, lowestBi tMask, and revNat).

\subsection{The test suite of containers}

The first tests were added to containers in $2007,{ }^{12}$ in the form of a few regression tests for observed bugs. Three years later, Don Stewart added a comprehensive test suite using QuickCheck (Claessen \& Hughes, 2000) with 91\% code coverage, and reported that "[n]o bugs were found". ${ }^{13}$ This test suite helped to maintain a consistently high quality and very few bugs crept into released versions of the library. In fact, the only serious bug mentioned in the library's changelog - a completely broken implementation of Data. IntMap. restrictKeys - only occurred because the tests for restrictKeys were accidentally not run as part of the test suite. ${ }^{14}$

\section{Overview of our verification approach}

In order to verify Set, IntSet, and Map, we use hs-to-coq to translate the unmodified Haskell modules to Gallina and then use Coq to verify the translated code. For example, consider the excerpt of the implementation of Set in Figure 1. The hs-to-coq tool translates this input to the following Coq definitions. ${ }^{15}$ The type name Set is renamed to Set_ to avoid clashing with the Coq keyword.

\footnotetext{
8 https://github.com/haskell/containers/commit/3535fcbe

9 https://github.com/haskell/containers/commit/d17d7182

10 https://github.com/haskell/containers/commit/c3083cfc

11 https://github.com/haskell/containers/pull/3

12 https://github.com/haskell/containers/commit/9d6c49b5

13 https://github.com/haskell/containers/commit/38743e39

14 https://github.com/haskell/containers/issues/392

15 In the file examples/containers/lib/Data/Set/Internal.v.
} 


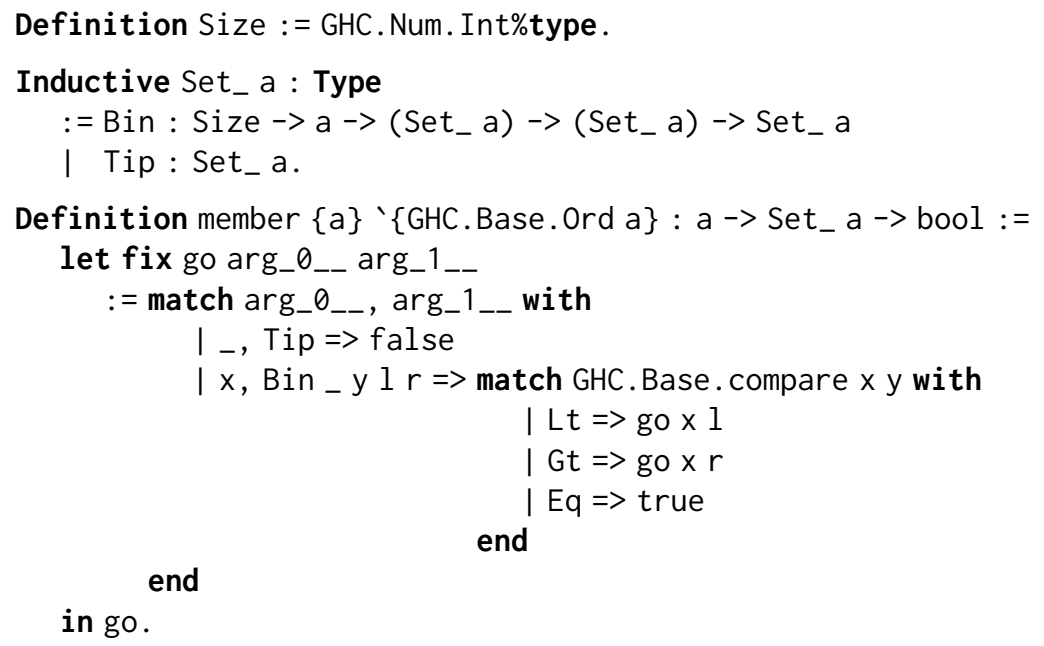

The unpacking and strictness annotations were ignored, as they do not make sense in Coq: unpacking only controls memory layout, and not the semantics of the type; and strictness annotations control evaluation order, which is immaterial in a total language. While in Haskell, the strictness annotations further rule out the use of infinite data structures, hs-to-coq already assumes that all data types are inductive (Spector-Zabusky et al., 2018), so they add nothing.

These definitions also depend on hs-to-coq's preexisting translated version of GHC's standard library base. Here, we use the existing translation of Haskell's Int type, the Ord type class, and Ord's compare method.

We carry out this translation for the Set, IntSet, and Map along with their attendant functions, and then verify the resulting Gallina code. In Section 4, we discuss the properties that we prove about these data structures, focusing on the details of the two set data structures. Naturally, this translation process means that these proofs comes with an attendant formalization gap, which we discuss in Section 7.1.

To further test the translation from Haskell to Coq, we also used Coq's extraction mechanism to translate the generated Gallina code, like that seen above, back to Haskell. This process converts the implicitly passed type-class dictionaries to ordinary explicitly passed function arguments, but otherwise preserves the structure of the code. For example, the member function above is extracted back to Haskell as the following code:

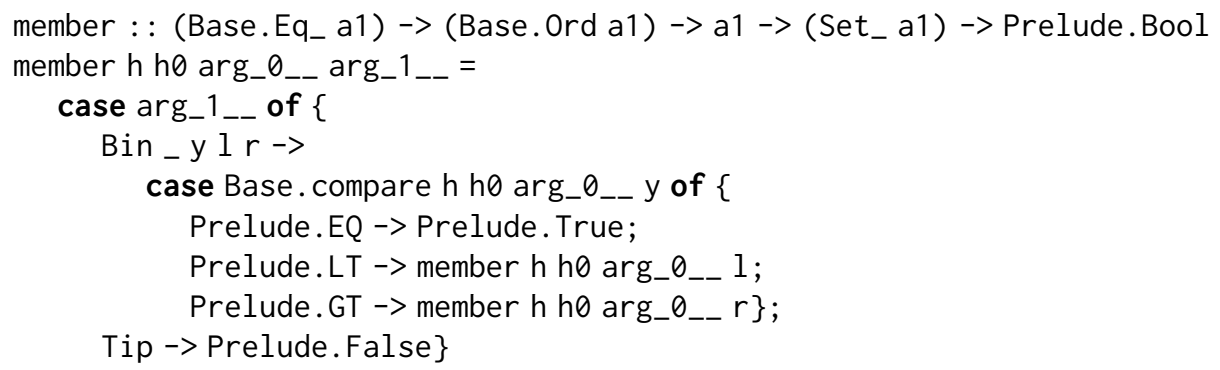

By providing an interface that restores the type-class-based types, we can run the original containers test suite against this code. This process helps us check that hs-to-coq 


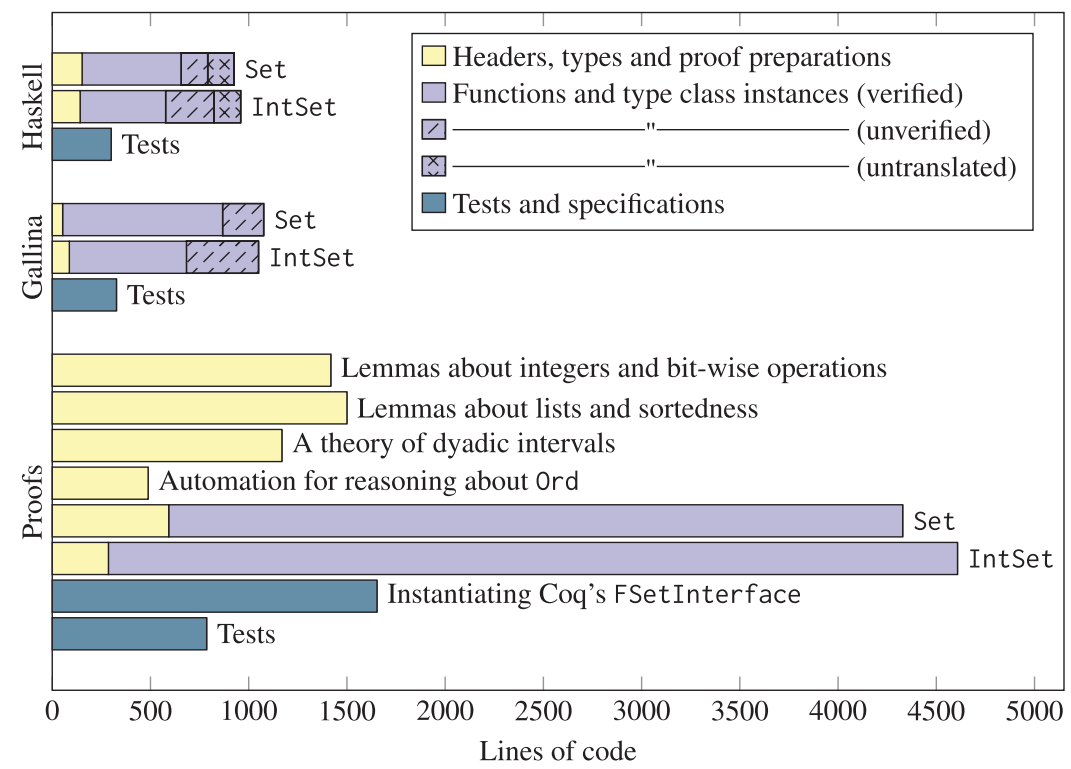

Fig. 3. A quantitative overview of the Haskell code, its translation into Coq and our proofs.

preserves the semantics of the original Haskell program during the translation process. Our handwritten shim module imports the extracted definitions above and then re-exports these definitions with their original types by using Haskell's type-class resolution to provide the explicit dictionaries.

- Create dictionary from an Eq type class constraint

eq_a : : Eq a $=>$ Base.Eq_a

eq_a $f=f$ (Base.Eq___Dict_Build $(==)(/=))$

- Create dictionary from an Ord type class constraint

ord_a : : Prelude.Ord a => Base. Ord a

ord_a _ = Base.ord_default Prelude. compare eq_a

-Restore the original type of the extracted member function -by providing explicit dictionaries

member : : Prelude.Ord a $\Rightarrow>$ a $\rightarrow$ Set a $\rightarrow$ Bool

member $=$ S2. member eq_a ord_a

Figure 3 provides an overview of the Haskell code that we target, the Gallina code that we translate it into, and the Coq proofs that we wrote. For ease of comparison (Map is quite different and much larger), the bar chart focuses on the two different set modules, but our numbers include Map. The relevant modules of the containers library contain 325 functions and 41 type class instances, written in 4096 lines of code (excluding comments and blank lines). Out of these, 36 functions and 20 type class instances (509 loc) were deemed "out of scope" and not translated. (We discuss untranslated definitions in more detail in Sections 5.3 and 7.2.) Our translation produces 4812 lines of Gallina code.

The Set, IntSet, and Map data structures come with extensive APIs. We specify and verify a representative subset of commonly used functions (listed in Figure 4), covering 
Set: API: delete, deleteMax, deleteMin, difference, disjoint, drop, elems, empty, filter, foldl, foldl', foldr, foldr', fromAscList, fromDescList, fromDistinctAscList, fromDistinctDescList, fromList, insert, intersection, isSubsetOf, lookupMax, lookupMin, map, mapMonotonic, maxView, member, minView, notMember, null, partition, singleton, size, split, splitAt, splitMember, take, toAscList, toDescList, toList, union, unions

Instances: Eq, Eq1, Monoid, Ord, Ord1, Semigroup

Internal functions: balanceL, balanceR, combineEq, glue, insertMax, insertMin, insertR, link, maxViewSure, merge, minViewSure, valid

IntSet: API: delete, difference, disjoint, elems, empty, filter, foldl, foldr, fromList, insert, intersection, isProperSubsetOf, isSubset0f, map, member, notMember, null, partition, singleton, size, split, splitMember, toAscList, toDescList, tolist, union, unions

Instances: Eq, Monoid, Ord, Semigroup

Internal functions: branchMask, equal, highestBitMask, mask, nequal, nomatch, revNat, shorter, valid, zero

Map: API: adjust, alter, assocs, balance, balanceL, balanceR, delete, deleteMax, deleteMin, difference, drop, elems, empty, filter, findIndex, findWithDefault, foldl, foldl', foldlWithKey', foldr, foldr', foldrWithKey, fromAscList, fromDescList, fromDistinctAscList, fromDistinctDescList, fromList, fromSet, glue, insert, insertLookupWithKey, insertMax, insertWith, insertWithKey, intersection, keys, keysSet, link, link2, lookup, lookupLE, lookupLT, lookupMax, lookupMin, mapEi ther, mapEi therWi thKey, mapMaybe, mapMaybeWi thKey, mapWi thKey, maxView, member, minView, notMember, null, partition, restrictKeys, singleton, size, split, splitAt, splitLookup, splitRoot, take, toAscList, toDescList, toList, union, unionWithKey, unions, unionsWith, update, updateLookupWi thKey, updateWi thKey, withoutKeys

Instances: Eq, Eq1, Monoid, Semigroup

Internal functions: insertMin, insertR, insertWi thKeyR, insertWithR, isProperSubmapOfBy, maxViewSure, minViewSure, splitMember

Fig. 4. The verified subset of functions and type classes in Data.Set, Data.IntSet, and Data.Map.Strict.

$68 \%$ of the Set API, $49 \%$ of the IntSet API, and $44 \%$ of the Map API. This verified API is complete enough to instantiate Coq's specification of finite sets and maps, along with many other specifications at varying levels of abstraction; for more details, see Section 4.

As Coq is not an automated theorem prover, verification of these complex data structures requires significant effort. In total, we verified 2331 lines of Haskell; the verification of Set, IntSet, and Map required 8.9 lines of proof per lines of code. This factor is noticeably higher for IntSet $(9.9 \times)$ than for Set $(7.4 \times)$, as the latter is conceptually simpler to reason about and allowed us to achieve a higher degree of automation using Coq tactics. 


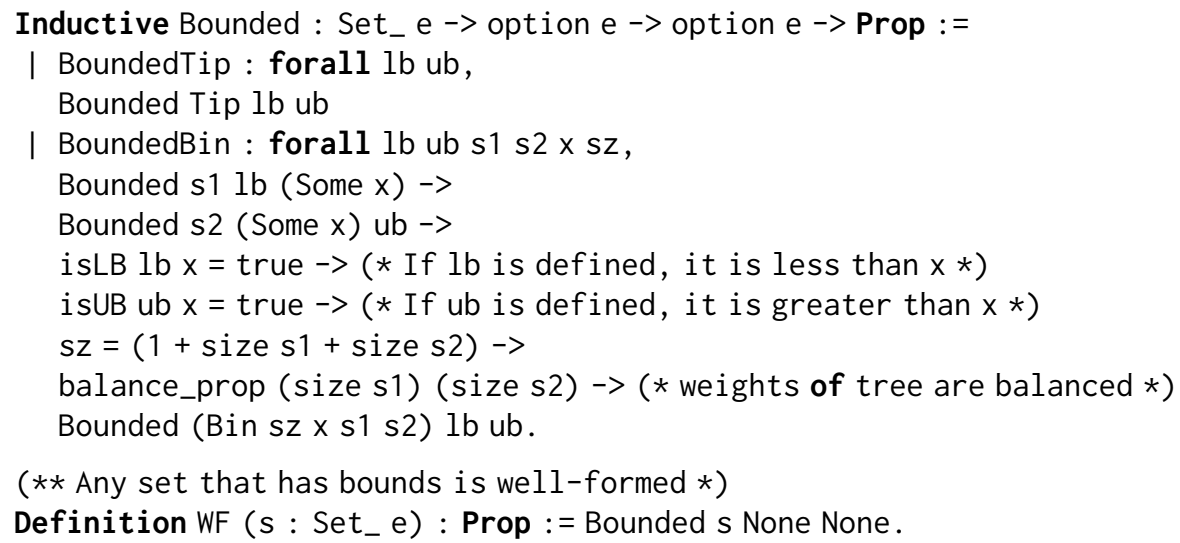

Fig. 5. Well-formed weight-balanced sets.

Our proofs also require the formalization of several background theories (not counted in the proof-to-code ratio above), including integer arithmetic and bits (1265 loc): lists and sortedness (1500 loc); dyadic intervals, which are used for verifying IntSet (1169 loc); and support for working with lawful Ord instances (488 loc), including a complete decision procedure, ${ }^{16}$ a port of Coq's Coq. Structures.OrdersTac to a setting using type classes instead of modules.

\section{Specifying Set and IntSet}

The phrase "we have verified this piece of software" on its own is meaningless: the particular specification that a piece of software is verified against matters. Good specifications are rich, two-sided, formal, and live (Appel et al., 2017). A specification is rich if it "describ[es] complex behaviors in detail". It is two-sided if it is "connected to both implementations and clients". It is formal if it is "written in a mathematical notation with clear semantics". And it is live if it is "connected via machine-checkable proofs to the implementation".

All specifications of Set and IntSet that we use are formal and live by definition. They are formal because we express the desired properties using Gallina, the language of the Coq proof assistant; and they are live because we use hs-to-coq to automatically convert containers to Coq where we develop and check our proofs. ${ }^{17}$ But how can we ensure that our specifications of Set and IntSet are two-sided and rich? How do we know that the specifications are not just facts that happen to be true, but are useful for the verification of larger systems? What complex behaviors of the data structures should we specify?

To ensure that our specifications are two-sided, we use specifications that we did not invent ourselves. Instead, we draw our specifications from a variety of diverse sources:

16 In the file examples/containers/theories/OrdTactic.v.

17 "Liveness" here solely refers to the fact that the specification and the Haskell source are automatically connected; it does not guarantee that the semantics of the two are identical. We discuss the formalization gap further in Section 7.1. 
from several parts of the containers codebase (Sections 4.1 to 4.4), from Haskell type class laws (Section 4.5), from preexisting Coq theories (Section 4.6), and from a mathematical description of sets (Section 4.7). This way, we also ensure that our specifications are rich because they describe the complex Set and IntSet data structures at varying levels of abstraction. Finally, by verifying the code against these disparate specifications, we not only increase the assurance that we captured all the important behaviors of Set and IntSet, but we also cross-validate the specifications against each other.

While we focus here on Set and IntSet, these techniques are all more general - in Section 5.9, we discuss how we were able to take the techniques presented here and extend them directly to verify the related Map data structure.

\subsection{Specifying implementation invariants}

Set and IntSet are two examples of abstract types whose correctness depend on invariants. Therefore, we define well-formedness predicates WF : Set_e -> Prop and WF : IntSet -> Prop and show that the operations preserve these properties. The definition of well-formedness differs between the two types, but specifications of both are already present within containers.

Well-formed weight-balanced trees. Our definition of WF for Set is derived from the valid function defined in the containers library. This function checks whether the input (1) is a balanced tree, (2) is an ordered tree, and (3) has the correct values in its size fields. It is not part of the normal, user-facing API of containers (since all exported functions preserve well-formedness), but is used internally by the developers for debugging and testing. For us, it is valuable as an executable specification, with less room for ambiguity and interpretation than comments and documentation.

However, rather than using valid directly, we define well-formedness as an inductive predicate, because we find it more useful from a proof engineering perspective. In particular, our definition of WF, as shown in Figure 5, relies on the Bounded inductive family. Its indices express lower and upper bounds of the elements stored in the tree; None means unbounded. At the same time, the property also checks that the sizes of the two subtrees are balanced in the Bin case. ${ }^{18}$ Nevertheless, we can relate the WF predicate to the valid function found in containers:

Lemma Bounded_iff_valid : forall s, WF $s<->$ valid $s=$ true.

Well-formed Patricia trees. Our well-formedness definition for IntSet is derived from the comments in the IntSet data type, shown in Figure 2, where the type declaration almost disappears beneath a large swath of comments describing its invariants.

In this case, the documentation-derived well-formedness predicate is stronger than the corresponding valid function from the implementation - the Haskell function was missing some necessary conditions. We reported this to the library authors, ${ }^{19}$ who have since fixed valid.

18 In the file examples/containers/theories/SetProofs.v.

19 https://github.com/haskell/containers/issues/522 
This fix to valid is an example of how verification allows us to cross-validate specifications and ensure that the invariants written in the comments are adequately reflected by the code. On the other hand, sometimes we discover that it is the comments in the code that are incomplete. For example, the comment describing the precondition for balanceL in the Data. Set module was misleading and too vague; for more details, see Section 6.1.

\subsection{Property-based testing}

At the next level of verification, we would like to show that the implementations of Set and IntSet are correct according to the implementors of the module. We specify correctness by deriving a definition directly from the test suite that is distributed with the containers library.

Thanks to the popularity of property-based testing within the Haskell community, this test suite contains a wealth of precisely specified general properties expressed using QuickCheck (Claessen \& Hughes, 2000). For example, one such property states that the union operation is associative, in terms of the Haskell-level equality function ==:

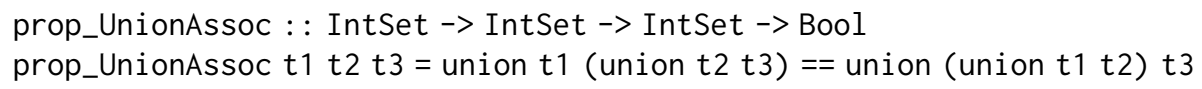

which we can interpret as a theorem about union: ${ }^{20}$

Theorem thm_UnionAssoc:

forall $t 1$, WF $t 1->$ forall $t 2$, WF $t 2->$ forall $t 3$, WF $t 3->$

union $t 1$ (union $t 2 t 3$ ) $==$ union (union $t 1 t 2$ ) $t 3=$ true.

We do not have to write these theorems by hand: as we describe in Section 5.8, we use hs-to-coq in a nonstandard way to automatically turn these executable tests into Gallina propositions (i.e., types). We have translated IntSet's test suite in this manner and have used our other specifications to prove that all QuickCheck properties about verified IntSet functions are theorems (with one exception due to our choice of integer representation see Section 5.5).

\subsection{Numeric overflow in Set}

There is one way in which we have diverged from the specification of correctness given by the comments of the containers library. The Data. Set module states: ${ }^{21}$

Warning: The size of the set must not exceed maxBound: : Int. Violation of this condition is not detected and if the size limit is exceeded, its behavior is undefined.

In practice, it makes no difference whether Int is bounded or not, as a valid set with $\left(2^{63}-1\right)$ elements would require at least 368 exabytes of storage. What does this imply for our specification of Set? Should we use fixed-width integers to represent Int? This choice would closely match the implementation, but we would have to carefully add preconditions to all our lemmas to avoid integer overflow, greatly complicating the proofs, with little

20 In the file examples/containers/theories/IntSetPropertyProofs.v.

21 http://hackage. haskell.org/package/containers-0.5.11.0/docs/Data-Set.html 
verification insight to be gained. Furthermore, such a specification would be difficult to use by clients, who themselves would need to prove that they satisfy such preconditions.

Instead, we translate Haskell's Int type to Coq's type of unbounded integers (called Z). This mapping avoids the problem of integer overflow altogether and is arguably consistent with the comment, as this choice replaces undefined behavior with concrete behavior. (The situation is slightly different for IntSet; see Section 5.5.)

\subsection{Rewrite rules}

So far, we have only been concerned with specifying the correctness of the data structures using the definition of correctness that is present in the original source code; the comments, the valid functions and the QuickCheck properties. However, to be able to claim that our specifications are two-sided, we need to show that the properties that we prove are useful to clients of the module.

One source of such properties are the rewrite rules (Peyton Jones et al., 2001) that enable library-specific compiler optimization. The containers library includes a small number of such rules. These annotations instruct the compiler to replace any occurrence of the pattern on the left-hand side in the rule by the expression on the right-hand side. The standard example is

$$
\text { \{-\# RULES "map/map" forall f g xs. map } f(\operatorname{map} g \times s)=\operatorname{map}(f . g) \times s ~ \#-\}
$$

which fuses two adjacent calls to map into one, eliminating the intermediate list.

The program transformation list fusion (Peyton Jones et al., 2001) is implemented completely in terms of rewrite rules, and the rules in the containers library set up its functions for fusion; an example is

\section{\{-\# RULES "Set. toAsclist"}

forall s. toAscList $s=$ build $(\backslash c n->$ foldrFB $c n s) \#-\}$

which transforms the toAscList function into an equivalent representation in terms of build.

We can view these rewrite rules as a direct specification of properties that the compiler assumes are true during compilation. Rewrite rules are used by GHC during optimization; if any of these properties are actually false, GHC will silently produce incorrect code. Therefore, any proof about the correctness of GHC's compilation of these files depends on a proof of these properties. We have manually translated all the rules into Coq - there are only few of them, so manual translation is viable - and have proved that the translated operations satisfy this specification. ${ }^{22}$

\subsection{Type classes with laws}

Many Haskell type classes come with laws that all instances of the type class should satisfy, which provides another source of external specification that we can use. For example, an instance of Eq is expected to implement an equivalence relation, an instance of Ord should describe a linear order, and an instance of Monoid should be, well, a monoid.

22 In the files examples/containers/theories/SetProofs.v and examples/containers/theories/IntSetProofs.v. 


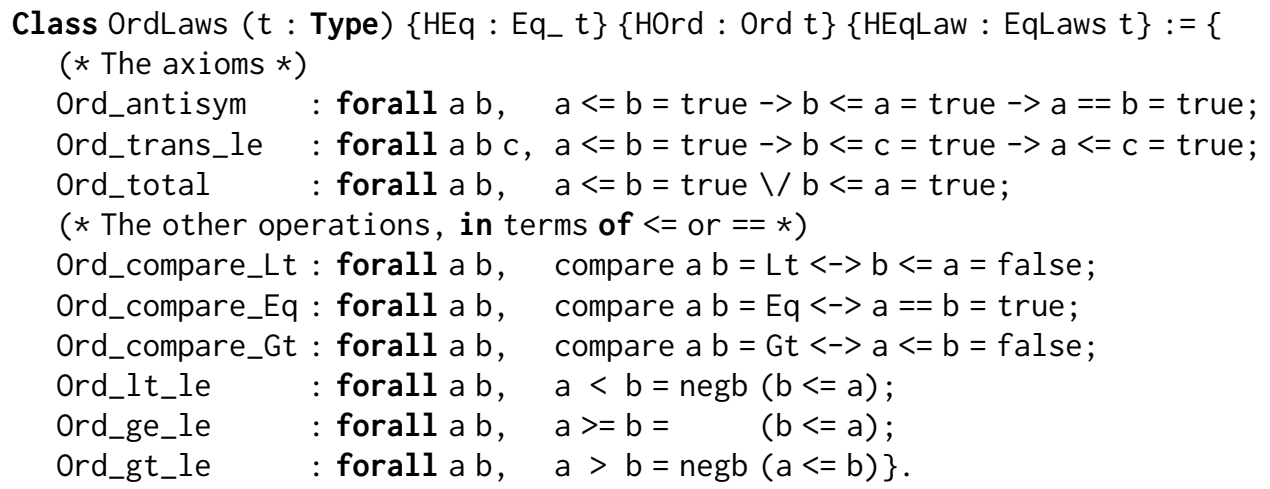

Fig. 6. Our codification of the Ord type class laws.

We reflect these laws using type classes whose members are the required properties. For example, we have defined EqLaws, OrdLaws (shown in Figure 6), and MonoidLaws. These classes can only be instantiated if the corresponding instance is lawful.

Even though we have defined these laws ourselves, using our understanding of what they mean for the Haskell standard library, we argue that they form a two-sided specification. In particular, we have been clients to the Ord laws in our verification of the Set data structure. Almost all theorems about Set must constrain the element type to one that is an instance of OrdLaws. Therefore, we know our specification of these laws is sufficiently strong to verify this library.

At the same time, we have also shown that multiple type class instances satisfy these laws including both set modules ${ }^{23}$ and other types such as Z, unit, tuples, option, and list. ${ }^{24}$ Because we successfully instantiated these type classes for many types, we also know that they are not too strong.

That said, nailing down the precise form of the type class laws can be tricky. Consider the case of a Monoid instance for a type T. The associativity law can be stated as "for all elements $x, y$ and $z$ of $T$, we have that $x<>(y<>z)$ is equal to $(x<>y)<>z$," where $<>$ is the monoid operation (i.e., mappend in Haskell). But in order to write this down as part of MonoidLaws in Coq, we need to make two decisions:

1. What do we mean by "equal"? The first option is to use Coq's propositional equality and require that $x<>(y<>z)=(x<>y)<>z$. This would, however, prevent us from making Set_, with $(<>)=$ union, a member of MonoidLaws: two extensionally equal sets may be represented by differently structured trees. Therefore, we instead require that the two expressions are equal according to their Eq instance: $(x$ $<>(y<>z)==(x<>y)<>z)=$ true. The tradeoff with this approach is that it precludes instances like MonoidLaws $b \rightarrow$ MonoidLaws $(a->b)$, since functions have no instance of Eq (and indeed, in general cannot have decidable equality).

For many types, however, this distinction is moot, since Haskell's equality coincides with structural equality; for example, this is the case for Bool, for Integer, and

23 In the files examples/containers/theories/SetProofs.v and examples/containers/theories/IntSetProofs.v.

24 In the file base-thy/GHC/Base.v. 
for IntSet itself (although not for Set, as mentioned above). To facilitate reasoning about such types, we provide the type class EqExact, which states that

forall $\{a\}$ a $\{$ EqExact $a\}, x==y=$ true $<->x=y$

2. What do we mean by "For all elements of T"? The obvious choice is universal quantification over all elements of $\mathrm{T}$ :

forall $(x$ y $z: T), x<>(y<>z)==(x<>y)<>z=$ true

But again, this collides with common practice in Haskell. Once again, consider Set: union only works correctly on well-formed sets. Therefore, our approach is to define an instance of this and other classes not for the type Set_e, but for the type of well-formed sets, $\{$ s : Set_e | WF s\}, where type class laws hold universally. This instance reflects the "external view" of the data structure - clients should only have access to well-formed sets.

An alternative could be to instead constrain MonoidLaws's theorems to hold only on members of $\mathrm{T}$ that are well-formed in some general way (e.g., according to an IsWF type class that could be instantiated at different types). In this way, we could instantiate MonoidLaws directly with types that require well-formedness, without the need for subset types.

\subsection{Specifications from the Coq standard library}

Because we are working in Coq, we have access to a standard library of specifications for finite sets, which we know are two-sided because they have already appeared in larger Coq developments. The Coq. FSets. FSetInterface module ${ }^{25}$ provides module types that cover many common operations and their properties. The module types come in two varieties: one that specifies sets of elements that merely have decidable equality (WSfun, WS), and one that specifies sets of elements that can be linearly ordered (Sfun, S). The WSfun and Sfun modules are presented as module functors that take an OrderedType module, containing the linearly ordered element type, as an input; the WS and S modules are the same, but they inline this information.

For example, the parameterized module type WSfun provides one specification of a finite set type, called $t$ in the excerpt from this interface below. The element type of this set, $E . t$, is required to have decidable equality.

Module Type WSfun (E : DecidableType).

Definition elt $:=E . t$.

Parameter $t:$ Type. (* Set type $*$ )

Parameter In : elt $->t \rightarrow$ Prop. (* Characteristic function for a Set $*$ )

Parameter mem : elt $\rightarrow t->$ bool. (* Membership function $*$ )

25 https://coq.inria.fr/library/Coq. FSets. FSetInterface.html 
(* Specification of mem *)

Parameter mem_1 : forall $\times$ s, In $\times$ s $\rightarrow$ mem $\times$ s $=$ true.

Parameter mem_2 : forall $\times \mathrm{s}$, mem $\times \mathrm{s}=$ true $\rightarrow$ In $\times \mathrm{s}$.

\section{End WSFun.}

Every operation in this interface, such as mem above, is accompanied by a small number of properties that specify the behavior of the operation.

We instantiate all four interfaces for Set and IntSet. ${ }^{26}$ For example, the instance for Set starts out:

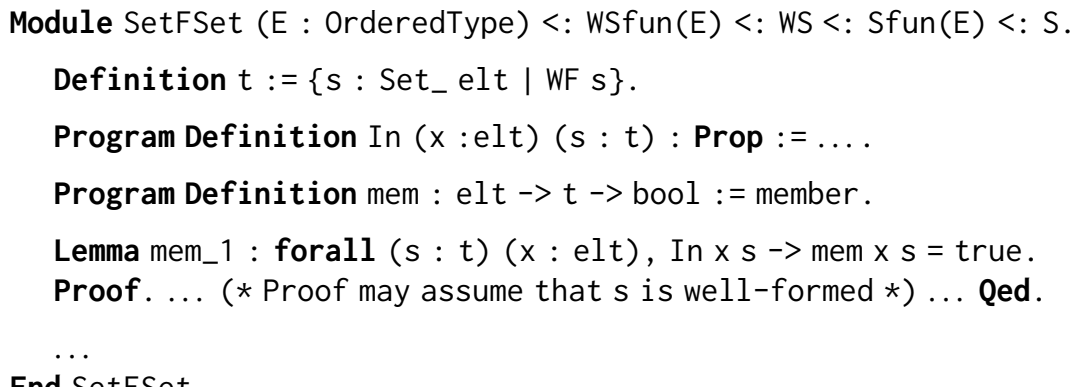

Instantiating these interfaces runs into two small hiccups. The first is that they talk about all sets, not simply all well-formed sets. Therefore, as in the previous section, we instantiate these interfaces with the subset type $\{\mathrm{s}:$ Set_ e | WF $s\}$. The second is that Coq's module system does not interact with type classes, and Set_ is defined such that its element type must be an instance of the Ord type class. This impedance mismatch requires us to write a module which can generate an Ord instance from a Coq OrderedType module.

By successfully instantiating this module interface, we obtain two benefits. First, we must prove theorems that cover many of the main functions provided by containers; these theorems are particularly valuable, as the interface itself is heavily used by Coq users. Second, by instantiating this interface, we connect our injected Haskell code to the Coq ecosystem, enabling Coq users to easily use the containers-derived data structures in their developments, should they so desire.

\subsection{Abstract models as specifications}

Tests, type classes, and the other sources of specifications do not fully describe the intended behavior of all functions. We, therefore, have to also come up with specifications on our own. We do this by relating a concrete search tree to the abstract set that it represents; that is, we provide a denotational semantics. We denote a set with elements of type e as its indicator function of type e $\rightarrow$ bool; for Set e, we provide a denotation function sem : forall $\{e\}$ ' $\left\{\mathrm{Eq}_{-} \mathrm{e}\right\}$, Set_e $->(\mathrm{e}->$ bool), and for IntSet, we provide a denotation relation Sem : IntSet $\rightarrow(\mathrm{N}->$ bool $) \rightarrow$ Prop.

\footnotetext{
26 In the files examples/containers/theories/SetProofs.v and examples/containers/theories/IntSetProofs.v.
} 
This approach allows us to abstractly describe the meaning of operations like insert. For Set_, we do this by providing a theorem like

Theorem insert_sem:

forall $\{a\}$ ' $\{0 r d L a w s ~ a\}$ (s : Set_a) $(x: a)$, WF $s \rightarrow$

forall ( $i: a)$, sem (insert $x s) i=(i==x)||$ sem $s i$.

(For IntSet, some technical details differ.) However, there is more we need to know about insert than just its denotation. We also need to know that it preserves well-formedness and bounds, and - to reason about balancing - its size. To avoid having to prove these properties independently, we define a relation Desc that completely describes a set, by asserting that it is well-formed and relating it to its bounds, its size and its denotation:

Definition Desc (s : Set_e) (lb ub : option e) (sz: Z) (f: e ->bool) := Bounded s lb ub 八 size s = sz 八 (forall $i$, sem $s i=f i)$.

This allows us to state a single theorem about insert, namely

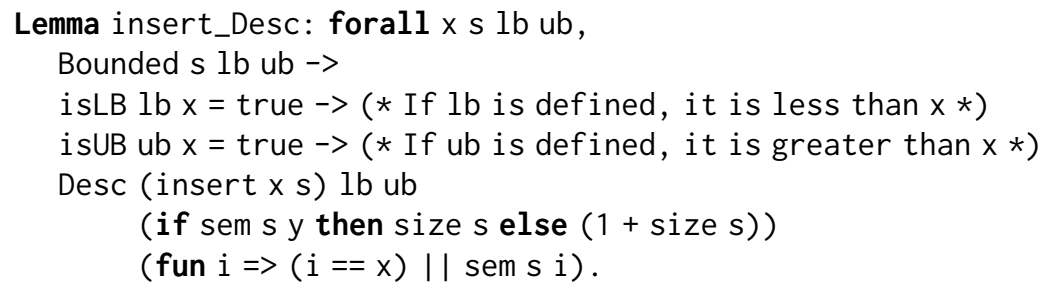

and prove everything we need to know about insert in one single inductive proof. This theorem describes insert completely: it describes its bounds, its size, its semantics, as well as its well-formedness. ${ }^{27}$

The theorem also introduces a layer of abstraction that we can build upon. In fact, we specify all functions this way, and use these specifications, rather than the concrete implementation, to prove the other specifications. (We have an analogous Desc relation for IntSet that describes the properties of Patricia trees.)

An alternative abstract model for finite sets is the sorted list of their elements, i.e. the result of toAscList. The meaning of certain operations, like foldr, take or size, can naturally be expressed in terms of toAscList, but would be very convoluted to state in terms of the indicator function, and we use this denotation - or both - where appropriate.

\section{Producing verifiable code with hs-to-coq}

Identifying what to prove about the code is only half of the challenge - we also need to get the Haskell code into Coq. Ideally, the translation of Haskell code into Gallina using hs-to-coq would be completely automatic and produce code that can be verified as easily as code written directly in Coq - and for textbook-level examples, that is the case (SpectorZabusky et al., 2018). However, working with real-world code requires adjustments to the translation process to make sure that the output is both accepted by Coq and amenable to verification.

27 In the file examples/containers/theories/SetProofs.v. 
A core principle of our approach is that the Haskell source code does not need to be modified in order to be verified. This principle ensures that we verify "the" containers library (not a "verified fork") and that the verification can be ported to a newer version of the library.

The crucial feature of hs-to-coq that enables this approach is the support for edits: instructions to treat some code differently during translation. Edits are specified in plain text files, which also serve as a concise summary of our interventions. The hs-to-coq tool already supported many forms of edits; for example, specifying when names need to be changed, when parts of the module should be ignored or replaced by some other term, when we want to map Haskell types to existing Coq types, or when a recursive function definition needs an explicit termination proof. In the course of this work, we added new features to hs-to-coq - such as the ability to apply rewrite rules, to handle partiality and to defer termination proofs to the verification stage - and extended the provided base library.

In this section we demonstrate some of the challenges posed by translating real-world code, and show how hs-to-coq's flexibility allowed us to not only to overcome them, but also to facilitate subsequently proving the input correct.

\subsection{Unsafe pointer equality}

An example of a Haskell feature that we cannot expect to translate without intervention is unsafe pointer equality. GHC's runtime provides the scarily named function reallyUnsafePtrEquality\#, which the containers library wraps as ptrEq : : a $>$ a -> Bool. If this function returns True, then both arguments are represented in memory by the same pointer. If this function returns False, we know nothing - this function is underspecified and may return False even if the two pointers are equal. In fact, if containers is compiled with a non-GHC compiler, it will define ptrEq to always return False.

The ptrEq operation is used, for example, in Set. insert $x$ s: If ptrEq indicates that the subtree with $x$ inserted is just the original subtree, function skips the redundant rebalancing step - which enhances performance - and returns the original set rather than constructing a semantically equivalent copy - which increases sharing. Because ptrEq is only ever used for optimization such as this, its weak specification is safe - the worst that could happen if it returns False overzealously is that some extra work is done.

Coq does not provide any way of reasoning about memory, so when we use hs-to-coq, we must replace ptrEq with something else. But what?

One option is to replace ptrEq with a definition that always returns False, using the following edit:

replace Definition ptrEq : forall $\{a\}, a_{->} a_{->}$bool := fun _ _ $=>$false.

This allows us to proceed with translation and verification. However, the code in the True branch of an unsafe pointer equality test would be dead code in Coq, and our verification would miss bugs possibly lurking there.

The next option would be to keep ptrEq completey abstract, for example by defining it as an Axiom. This would indeed force us to consider both branches. Unfortunately, we will likey fail to conclude the True branch, as that code is written under the assumption that the pointers are indeed equal, and thus the values structurally equal. 
This leads us to the chosen solution, where we leave ptrEq unspecified, to force us to consider both branches, but in the True branch we obtain the additional assumption that the values are structurally equal, by assuming the implication ptrEq $x y=$ true $\rightarrow x=y$.

Instead of using the dreaded Axiom to state this, we can achieve the same by way of an opaque and partially defined definition of ptrEq: ${ }^{28}$

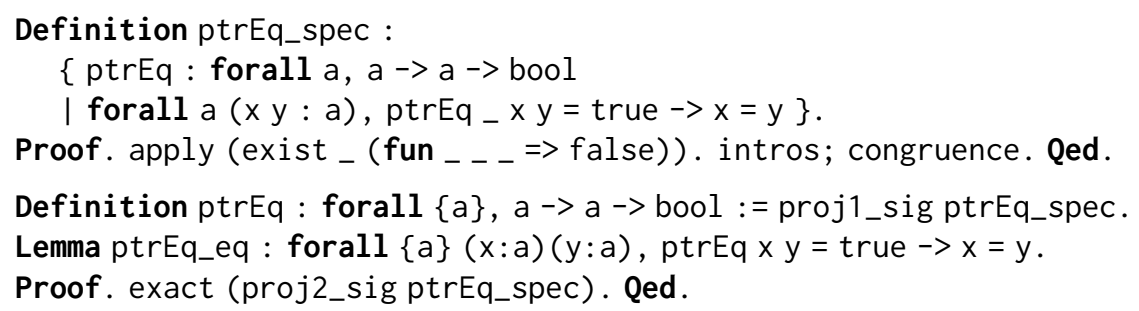

The Qed at the end of the definition of ptrEq_spec hides the concrete witness (which simply always returns false and thus satisfies the specification vacuously) and forces verification to proceed down both paths.

For idiomatic uses of ptrEq, where the True branch is some optimized variant of the False case, this solution is entirely satisfactory. But obviously this does not capture the behavior GHC's reallyUnsafePtrEquali ty\# completely, and creative use of ptrEq can exploit this gap. For example, the expression $x==y \& \&$ ptrEq y $z \& \&$ not ( $p \operatorname{trEq} x z$ ) evaluates to True in Haskell when $x$ and $z$ are the same pointers and $y$ is structurally equal but a different pointer, but can be proven to be always False in Coq, becuase in Coq ptrEq respects structural equality.

\subsection{Evaluation order}

A shallow embedding of Haskell into Coq makes the difference between strict and lazy code vanish, because Gallina is a total language and does not care about evaluation order.

Haskell has "magic" functions like seq that allow the programmer to explicitly control strictness, and the containers library uses it to improve performance. Its effect is irrelevant in Coq, and we instruct hs-to-coq to use this simple, magic-free implementation for it:

Definition seq $\{a\}\{b\}(x: a)(y: b):=y$.

\subsection{Eliminating unwanted parts of the code}

Figure 7 lists the untranslated portions of the Set, IntSet, and Map modules. This makes up $15 \%$ of the code, as can also be seen in Figure 3, and is smaller than the parts that were verified (see Figure 4).

Many of these operations are functions that we choose to ignore for the sake of verification - for example, the function showTree in Data. Set prints the internal structure of

28 In the file examples/containers/lib/Utils/Containers/Internal/PtrEquality.v. 
Set: API: deleteAt, deleteFindMax, deleteFindMin, elemAt, findIndex, findMax, findMin

Instances: Data, IsList, NFData, Read, Show, Show1

Internal functions: showTree

IntSet: API: deleteFindMax, deleteFindMin, findMax, findMin, fromAscList, fromDistinctAscList

Instances: Data, IsList, NFData, Read, Show

Internal functions: showTree

Map: API: alterF, atKeyImpl, deleteFindMax, deleteFindMin, differenceWith, differenceWi thKey, elemAt, findMax, findMin

Instances: Data, IsList, NFData, Ord1, Read, Read1, Show, Show1, Show2

Internal functions: , al terFCutoff, al terFFallback, al terFYoneda, bogus, deleteAlong, filterGt, filterLt, find, insertAlong, lookupTrace, replaceAlong

Fig. 7. Untranslated functions and type classes in Data. Set, Data. IntSet, and Data. Map. Strict.

such a set as an ASCII-art tree. This function is not used elsewhere in the module. In the interest of a tidier and smaller output, we skip this function using an edit:

skip showTree

Similarly, we skip functionality related to serialization (the Show and Show1 type classes), deserialization (the Read type class), generic programming (the Data type class), and overloaded list notation (the IsList type class).

Furthermore, we skip some operations whose public API is partial. For example, evaluating findMax empty will call error and throw an exception, as the empty set has no maximum element. We cannot model this exception in Coq (see Section 5.4 for how we handle calls to error within total functions), so we skip findMax and similar functions (findMin, deleteFindMax, deleteFindMin, findIndex, elemAt and deleteAt). This elision is not significant because the containers API provides total equivalents for many of these functions (e.g., lookupIndex, which returns Nothing when the index is out of bounds).

Finally, we skip the two functions that use mutual recursion as this feature is not yet supported by hs-to-coq (fromAscList and fromDistinctAscList). While basic structural mutual recursion is supported by Coq, the existing facilities for nonstructural recursion (such as Program Fixpoint; see Section 5.7) do not support mutual recursion.

\subsection{Partiality in total functions}

In contrast to the skipped functions above, some functions use partiality in their implementation in ways that cannot be triggered by a user of the public API. In particular, they may use calls to Haskell's error function when an invariant is violated.

For example, the central balancing functions for Sets, balanceL and balanceR, may call error when passed an ill-formed Set. Because our proofs only reason about 
well-formed sets, this code is actually dead. It does not matter how we translate error any term that is accepted by Coq is good enough. However, error in Haskell has the type

error : : String $\rightarrow$ a

which means that a call to error can inhabit any type. We cannot define such a function in Coq, and adding it as an axiom would be glaringly unsound.

Therefore, we extended hs-to-coq to use the following definition for error:

Class Default (a:Type) $:=\{$ default : a $\}$.

Definition error $\{a\}$ ' $\{$ Default $a\}$ : String $\rightarrow$ a.

Proof. exact (fun $=>$ default). Qed.

The type class enforces that we use error only at non-empty types, ensuring logical consistency. Yet we will notice that something is wrong when we have to prove something about it. Just as with ptrEq (Section 5.1), by making the definition of error opaque using Qed, we are prevented from accidentally or intentionally using the concrete default value of a given type in a proof about error. Furthermore, when we extract the Coq code back to Haskell for testing, we translate this definition back to Haskell's error function, preserving the original semantics.

This encoding is inspired by Isabelle, where all types are inhabited and there is a polymorphic term undefined : : a that denotes an unspecified element of any type.

\subsection{Translating the Int in IntSet}

As discussed in Section 4.1, we map Haskell's finite-width integer type Int to Coq's unbounded integer type $Z$ in the translation of Data. Set in order to match the specification that integer overflow is outside the scope of the specified behavior.

For IntSet, however, this choice would cause problems. Big-endian Patricia trees require that two different elements have a highest differing bit. This is not the case for $Z$, where negative numbers have an infinite number of bits set to 1 ; for instance, -1 is effectively an infinite sequence of set bits. Fortunately, hs-to-coq is flexible enough to allow us to make a different choice when translating IntSet; we can pick any suitable type where all elements have a finite number of bits set, such as the natural numbers $(\mathrm{N})$ or a fixed width integer type.

Given that Coq's standard library provides a fairly comprehensive library of lemmas about $\mathrm{N}$ and decision procedures (omega and $\mathrm{lia}$ ) that work with it, we chose to use $\mathrm{N}$ for now, with the intention to eventually switch to a 64-bit integer type. This is the appropriate generalization of IntSet to an infinite domain. In Haskell, the domain is 64-bit words, which happen to be interpretable as negative numbers. When we generalize to an infinite domain, we generalize to bit strings of unbounded but finite length, which we can most simply interpret as nonnegative.

The IntSet code uses bit-level operations, like complement and negate, that do not exist for N. To deal with this we extended hs-to-coq with support for rewrite edits like

rewrite forall xy, (x \&\&. complement $y)=($ xor $x(x . \& . y))$

which instruct it to replace any expression that matches the left-hand side by the righthand side. For signed or bounded integer types, both sides are equivalent. For unbounded 
unsigned types, like Coq's type $\mathrm{N}$, the left hand side is undefined (values in $\mathrm{N}$ have no complement in $\mathrm{N}$ ), while the right-hand side is perfectly fine. When we switch to bounded integers in the IntSet code, we can remove these edits.

\subsection{Low-level bit twiddling}

The containers library uses highly tuned bit-twiddling algorithms to operate on IntSets. For example, the function revNat reverses the order of the bits in a 64-bit number:

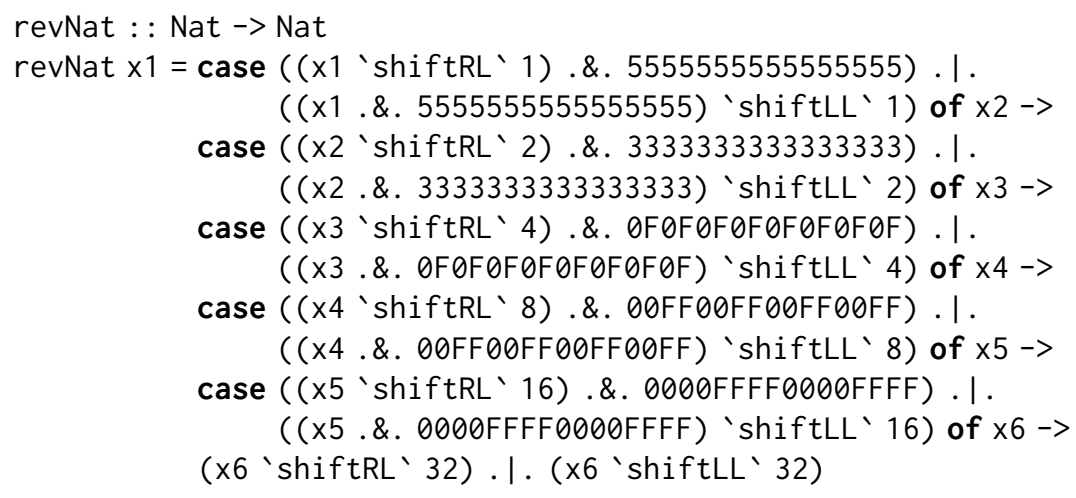

Though complicated, this code is within the scope of what hs-to-coq can translate, and we can verify its correctness. ${ }^{29}$

However, we can't keep up with all their tricks. For example, indexOfTheOnlyBit, which was contributed by Edward Kmett, ${ }^{30}$ takes a number with exactly one bit set and calculates the index of said bit. It does so by unboxing ${ }^{31}$ the input, multiplying it by a magic constant, and using the upper 6 bits of the product as an index into a table stored in an unboxed array literal. This manifests as the following scary-looking code:

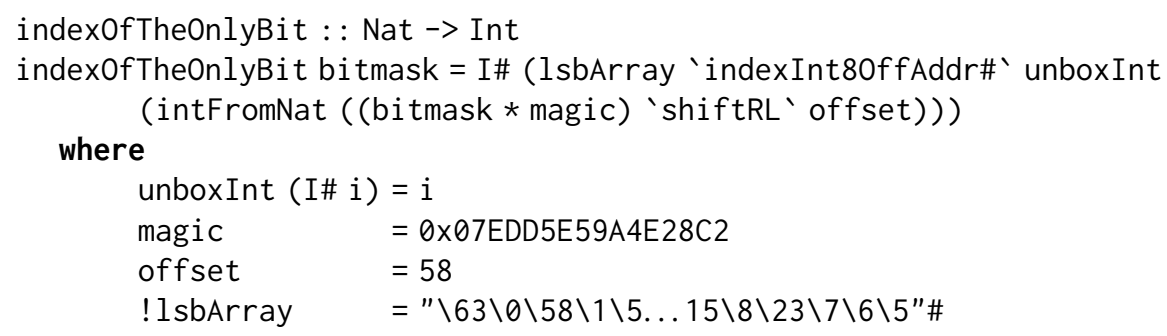

We currently cannot translate this code because hs-to-coq does not yet support unboxed arrays or unboxed integers. Even if we could (such as by treating the unboxed values as ordinary boxed values), the algorithm it uses depends crucially on the finite width of machine words, relying on both a finite lookup table (lsbArray) as well as on unsigned integer overflow in the multiplication. We therefore replace it with a simpler but

29 In the file examples/containers/theories/RevNatSlowProofs.v.

30 https://github.com/haskell/containers/commit/e076b33f

31 Unboxing is analogous to the $\{-\#$ UNPACK \#- $\}$ pragma mentioned in Section 2.2; unpacking a field stores an unboxed value there. Unboxed values are always fully evaluated, bypassing the uniform, lazy representation common to all other Haskell values. For example, unboxed Ints are machine words and may be stored in registers. 
more general definition based on a integer logarithm function provided by Coq's standard library:

redefine Definition indexOfTheOnlyBit := fun $x=>N . \log 2 x$.

Similarly, we provide simpler definitions for the four low-level bit-twiddling functions branchMask, mask, zero and suffixBitMask.

\subsection{Nontrivial recursion}

In order to prove the correctness of Set and IntSet, we must deal with termination. There are two reasons for this. First, we intrinsically want to prove that none of the functions provided by containers go into an infinite loop. Second, Coq requires that all defined functions are terminating, as unrestricted recursion would lead to logical inconsistencies. Depending on how involved the termination argument for a given function is, we use one of the following four approaches.

\subsubsection{Obvious structural recursion}

By default, hs-to-coq implements recursive functions directly using Coq's fix keyword. This works smoothly for primitive structural recursion; indeed, a majority of the recursive functions that we encountered, such as member in Figure 1, were of this form and required no further attention.

\subsubsection{Almost-structural recursion}

Another common recursion pattern can be found in binary operations such as link in Figure 8 . Here, every recursive call shrinks either or both of its arguments to immediate subterms of the originals, leaving the others unchanged. This almost-structural recursion is already beyond the capabilities of Coq's termination checker. We, therefore, instruct hs-to-coq to use Coq's Program Fixpoint command to translate these functions in terms of well-founded recursion by adding the edits

termination link \{measure (size_nat arg_o_- + size_nat arg_1__) \}

obligations link termination_by_omega

This specifies both:

1. The termination measure, which is the sum of the sizes of the arguments (we defined the function size_nat: IntSet $->$ nat).

2. The termination proof that the measure decreases on every call. This is represented as the Coq tactic termination_by_omega, which is a thin wrapper we defined around omega, a Coq tactic to decide linear integer arithmetic.

We can use these edits (with size_nat and termination_by_omega) to get Coq accepts such recursive definitions without the need for any further proofs or manual intervention.

\subsubsection{Well-founded recursion}

A small number of functions recurse in a nonstructural way, such as foldlBits in Figure 8, which recurses on the input after clearing the least-significant set bit (bm 'xor' 


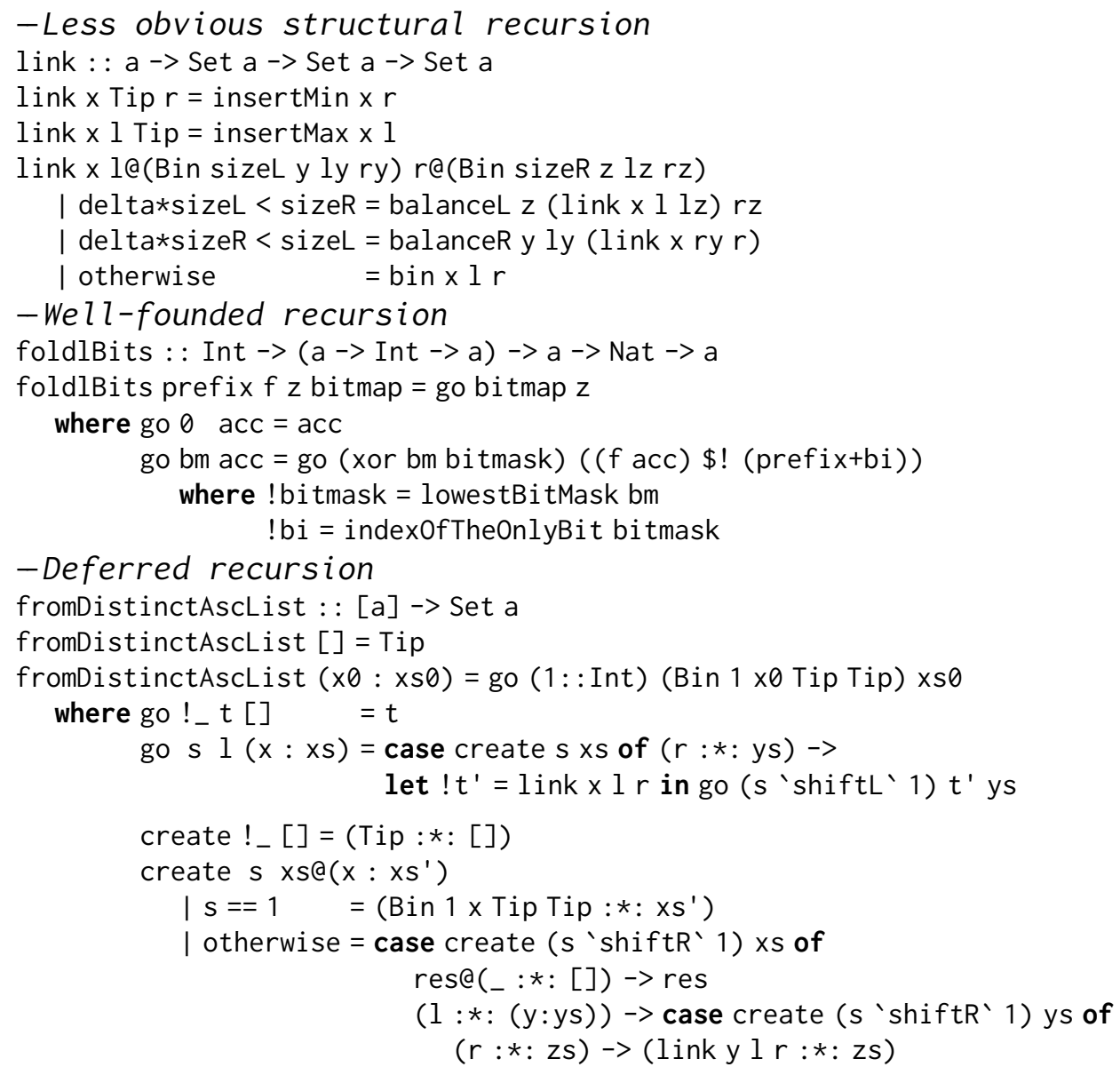

Fig. 8. Recursion styles.

(lowestBitMask bm)). We can handle this sort of logic using the same machinery as before, but now we have to write a specialized termination tactic and declare it in the obligations hint. To do so, we need to prove necessary lemmas before translating the Haskell module in question. In particular, our lemmas cannot mention functions that are defined in the translated Haskell module. We can do that by exploiting the fact that Coq's value definitions are not generative: since the function lowestBitMask is defined elsewhere to be lowestBitMask (bm: $N$ ) $=2{ }^{\wedge} N_{-}$ctz bm, we can still use lemmas such as foldlBits_proof to reason about lowestBitMask:

Lemma foldlBits_proof: forall a, N. eqb a $\theta=$ false $\rightarrow$ N.to_nat $\left(N .1\right.$ xor a $\left(2^{\wedge}\right.$ N_ctz $\left.\left.a\right)\right)<N$. to_nat a.

Coq's Program Fixpoint only supports top-level functions, but we frequently encounter local recursive functions - the go idiom, as seen here. To support this, we extended hs-to-coq to offer some of the convenience provided by Program Fixpoint 
by translating local recursive functions using the same well-founded-recursion-based fixed-point combinator as Program Fixpoint.

\subsubsection{Deferred recursion}

Finally, we encounter some functions that require elaborate termination arguments, such as fromDistinctAscList in Figure 8. It has two local recursive functions, go and create, and to convince ourselves that fromDistinctAscList is indeed terminating, we have to reason as follows:

The function create bitshifts its first argument to the right upon each recursive call, until the argument is 1. Ergo, it is terminating, but only for positive input - it clearly loops if $x$ is 0 . The function go recurses on smaller lists as its third argument, but to see that, we first have to convince ourselves that the list in the tuple returned by create is never larger than the list passed to create. Also, go calls create, so we need to ensure that the s passed to it is positive. The go function bitshifts $S$ to the left at every call, so if $g o$ is called with a positive $S$, then $S$ will remain positive in recursive calls. Finally, we see that fromDist inctAscL ist calls go with $s$ equal to 1 , which is positive, so we can conclude that fromDist inctAscL ist terminates.

If we wanted to convince Coq of this termination pattern, we would have to turn create and go into top-level definitions, change their types to rule out invalid (nonpositive) inputs, define create using Program Fixpoint, and provide an explicit termination argument by well-founded recursion. Then we could prove that create preserves the list lengths, which we need to define go, again using Program Fixpoint. This is certainly possible, but it is not simple, especially in an automatic translation.

For hard cases like these we resort to deferred termination checking, a feature that we added to hs-to-coq. We can instruct it to use the following axiom as a very permissive fixed-point combinator and translate the code of fromDistinctAscList essentially unchanged:

Axiom deferredFix :

forall $\{a r\}$ ' $\{$ Default $r\},((a->r) \rightarrow(a->r)) \rightarrow a->r$.

On its own, deferredFix does not do anything; it merely sits in the translated code applied to the original function body. It is consistent, since its type could be implemented by a function that always returns default (see Section 5.4). And it does not prevent the user from running extracted code - we can extract this axiom to the target language's unrestricted fixpoint operator (e.g., Data. Function. fix in Haskell), although this costs us the guarantee that the extracted code is terminating.

When it comes time to verifying something about a function that is defined using deferredFix, we need to give deferredFix meaning. We do so using a second axiom, deferredFix_eq_on, which states that for any well-founded relation R (well_founded $R$ ), if the recursive calls in $f$ are always at values that are strictly $R$-smaller than the input (recurses_on $R$ ), then we may unroll the fixpoint of $f$ :

Definition recurses_on $\{a b\}$

(P: $a->$ Prop) (R: a $\rightarrow$ a $\rightarrow$ Prop) $(f:(a->b) \rightarrow(a->b))$ 


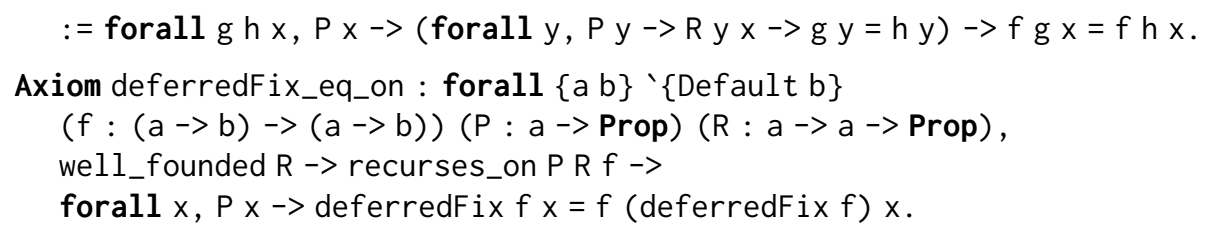

The predicate $P$ : a $\rightarrow$ Prop allows us to restrict the domain to inputs for which the function is actually terminating - crucial for go and create.

The predicate recurses_on P R $f$ characterizes the recursion pattern of $f$, but does so in a very extensional way and only considers recursive calls that can actually affect the result of the function. For instance, it would consider a recursive function defined by $f n=$ if $f$ $\mathrm{n}$ then true else true to be terminating.

We can use this nontrivial axiom without losing too much sleep, because we we have an implementation of deferredFix and deferredFix_eq_on in terms of classical logic and the axiom of choice (as provided by the Coq module Coq.Logic.Epsilon). ${ }^{32}$ This means that both axioms are consistent with plain Coq. We do not know if deferredFix_eq_on is strictly weaker than classical choice, so users of hs-to-coq who want to combine the output of hs-to-coq with developments known to be inconsistent with classical choice (e.g., homotopy type theory) should be cautious.

Pragmatically, working with deferredFix is quite convenient, as we can prove termination together with the other specifications about these functions. The actual termination proofs themselves are not fundamentally different from the proof obligations that Program Fixpoint would generate for us and - although not needed in this example - can be carried out even for nested recursion through higher order functions like map.

This approach to defining recursive functions via extensionality was inspired by Isabelle's function package (Krauss, 2006). It is also an instance of the recursion schemes described by Charguéraud (2010a).

\subsection{Translating Haskell tests to Coq types}

When we translate code, we usually want to preserve the semantics of the code as much as possible. Things are very different when we translate the QuickCheck tests defined in the containers test suite, as we discussed in Section 4.2: whereas, the semantics of the test suite in Haskell is a program that creates random input to use as input to test executable properties, we want to reinterpret these properties as logical propositions in Coq. Put differently, we are turning executable code into types.

Recall the example QuickCheck property that we saw Section 4.2, which specifies that set union is associative in terms of the Haskell-level equality function ==:

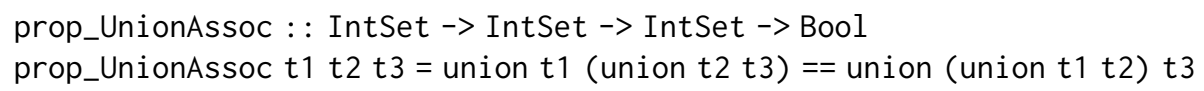

How does QuickCheck work with this?

QuickCheck's API provides types and type classes for writing property-based tests. In particular, it defines an opaque type Property that describes properties that can be checked

32 In the file base/GHC/DeferredFixImpl.v. 
using randomized testing, a type class Testable that converts various testable types into a Property, and a type constructor Gen that describes how to generate a random value. These are combined, for instance, in the QuickCheck combinator

forAll : : (Show a, Testable prop) $\Rightarrow$ Gen a $\rightarrow$ ( a -> prop) -> Property

which tests the result of a function on inputs generated by the given generator. The Testable instance for functions, for example, uses forAll to generate an input and then tests the output; testing prop_UnionAssoc thus involves, essentially, evaluating

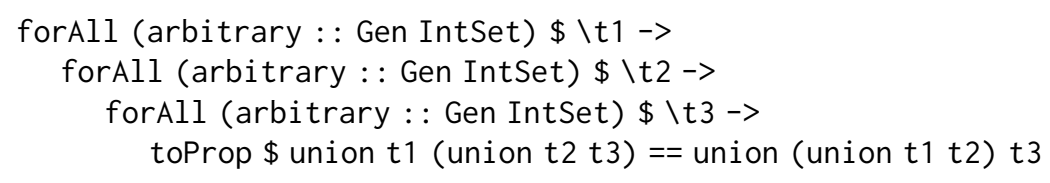

Since we want to prove, not test, these properties, we do not convert the QuickCheck implementation to Coq. Instead, we write a small Coq module that provides the necessary pieces of the interface of Test.QuickCheck, but interprets these types and functions in terms of Coq propositions. In particular:

- We use Coq's non-computational type of propositions, Prop, instead of using QuickCheck's computational type of results Property;

- Gen a is simply a wrapper around a logical predicate on a; and

- forAll quantifies (using Coq's forall) over the type a, and ensures that the given function - now a predicate - holds for all members of a that satisfy the given "generator".

Concretely, this leads to the following adapted Coq code:

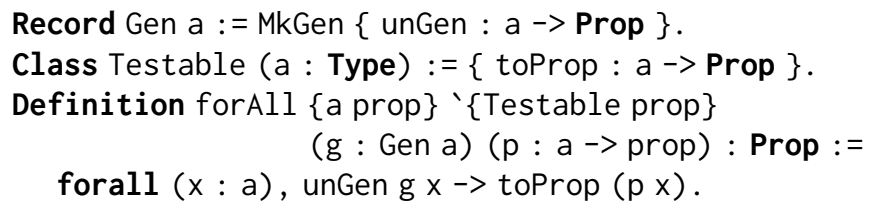

We provide similar translations for QuickCheck's operators ===, ==>, .\&\&. and .||., and we replace generators such as choose : : Random $a=>(a, a)->$ Gen a with their corresponding predicates.

With this module in place, hs-to-coq translates the test suite into a "proof suite". As we saw in Section 4.2, a test like prop_UnionAssoc is now a definition of a Coq proposition, that is to say a type, and can be used as the type of a theorem:

Theorem thm_UnionAssoc : toProp prop_UnionAssoc.

If we evaluate toProp, we get that this theorem equivalent to the following expanded form (which we saw back in Section 4.2):

Theorem thm_UnionAssoc:

forall $t 1$, WF $t 1->$ forall $t 2$, WF $t 2->$ forall $t 3$, WF $t 3->$ union $t 1$ (union $t 2 t 3$ ) $==$ union (union $t 1 t 2$ ) $t 3=$ true.

Here, the WF constraints correspond to the arbitrary generators in Haskell, which were constrained to only generate well-formed IntSets. 
API: adjust, alter, assocs, balance, balancel, balanceR, delete, deleteMax, deleteMin, difference, drop, elems, empty, filter, findIndex, findWithDefault, foldl, foldl', foldlWithKey', foldr, foldr', foldrWithKey, fromAscList, fromDescList, fromDistinctAscList, fromDistinctDescList, fromList, fromset, glue, insert, insertLookupWi thKey, insertMax, insertWith, insertWithKey, intersection, keys, keysSet, link, link2, lookup, lookupLE, lookupLT, lookupMax, lookupMin, mapEi ther, mapEi therWi thKey, mapMaybe, mapMaybeWi thKey, mapWithKey, maxView, member, minView, notMember, null, partition, restrictKeys, singleton, size, split, splitAt, splitLookup, splitRoot, take, toAscList, toDescList, toList, union, unionWithKey, unions, unionsWith, update, updateLookupWi thKey, updateWi thKey, wi thoutKeys

Instances: Eq, Eq1, Monoid, Semigroup

Internal functions: insertMin, insertR, insertWithKeyR, insertWithR, isProperSubmapOfBy, maxViewSure, minViewSure, splitMember

Fig. 9. Verified functions from Data.Map. Strict.

\subsection{Verifying Data.Map.Strict}

We also used hs-to-coq to verify large parts of Data.Map.Strict, a finite map implementation from the containers library. ${ }^{33}$ Since the underlying data structure - a weight-balanced tree - is the same as that of Data. Set, many of the specifications and proofs are broadly similar. As before, the specifications draw on type class laws, Coq's FMapInterface module, and an abstract definition similar to that of Section 4.7. The verified functions from this module are listed in Figure 9.

We used the function sem: forall $\{$ e a $\}$ ' $\left\{E_{-}\right.$e $\}$, Map e a $\rightarrow$ e $\rightarrow$ option a as the denotation for maps. This type introduces added complexity into the specification and proofs relative to the indicator function for sets, as we use an option a instead of simply a boolean value. When working with a Set, it is sufficient to prove that a given element exists somewhere in the set; but when working with a Map, we must show that a given key not only appears, but also is mapped to the correct value. For example, in fromList, the description specifies that if a key appears multiple times in the pairs of an input list, the last value in the list is the one that is used in the map.

Nevertheless, only slight modifications of the specifications and proofs developed for Data. Set (such as the Desc relation described in Section 4.7) were required to be able to handle this additional complexity. Furthermore, this machinery is also applicable to functions in the Data.Map.Strict API that have no analogue in Data. Set. These extensions provide further evidence for the utility and flexibility of the original specifications.

Our verification of Data.Map.Strict had one source of specification not available to Data.Set. In other work, we have experimented with the use of hs-to-coq in order to verify optimization passes in GHC's intermediate language, Core. ${ }^{34}$ These optimizations use finite maps to record variable sets and environments. Since we were verifying

33 The library comes in two varieties: Data.Map.Strict, which forces the evaluation of the values in the map; and Data.Map.Lazy, which does not. As we do not preserve evaluation order (as discussed in Section 5.2, the difference is not relevant to our work.

34 See the examples/ghc directory. 
these before we had verified Data.Map, during the verification process, we recorded the properties of the maps that were required as a list of over thirty axioms. For example, one axiom states that if a key-value pair is inserted in a map, then looking up the given key should return the given value. This property was expressed as the following:

Lemma lookup_insert: forall key value (m: Map e a),

WF $m \rightarrow$

lookup key (insert key value $\mathrm{m}$ ) = Some value.

In this work, we were able to prove all of these lemmas using our abstract Desc specifications in a straightforward way.

Lastly, the vast majority of the Data.Map.Strict proofs were completed by the sixth author, an undergraduate with limited Haskell and Coq experience. This both demonstrates the utility of the hs-to-coq tool and suggests that producing verified code is a realistic task even without significant expertise.

\section{Contributions to containers: Theory and practice}

We chose the Set, IntSet, and Map modules of the containers library as our target because they nicely represent the kind of Haskell code that we want to see verified in practice. Nevertheless, the deep understanding required to verify these modules led to new insights into the algorithms themselves and to improvements to their Haskell implementation.

\subsection{New insight into the verification of weight-balanced trees}

The data structure underlying Set and Map was originally presented by Nievergelt \& Reingold (1972). It is a binary search tree with the invariant that if $s_{1}$ is the size of the left subtree and $s_{2}$ the size of the right subtree of a node, then

$$
\operatorname{bal}_{\mathrm{NR}}\left(s_{1}, s_{2}\right):=\left(s_{1}+1\right) \leq \delta \cdot\left(s_{2}+1\right) \wedge\left(s_{2}+1\right) \leq \delta \cdot\left(s_{1}+1\right)
$$

holds for a balancing tuning parameter $\delta$. In 1992, Adams suggested a variant of the balancing condition, namely

$$
\operatorname{bal}\left(s_{1}, s_{2}\right):=s_{1}+s_{2} \leq 1 \vee\left(s_{1} \leq \delta \cdot s_{2} \wedge s_{2} \leq \delta \cdot s_{1}\right) .
$$

The conditions are very similar, but not equivalent: the former allows, for example, $\delta=3$, $s_{1}=2$ and $s_{2}=0$, which the latter rejects.

Initially, the containers library used Adams's balancing condition with the parameters $\delta=4$ (for sets) or $\delta=5$ (for maps). Campbell (2010) found that these parameters are actually invalid and exhibited a sequence of insertions and deletions that produced an unbalanced tree. Subsequently, the containers library switched to $\delta=3$ in both modules. Inspired by this bug report, Hirai \& Yamamoto (2011) thoroughly analyzed this data structure with the help of a Coq formalization, and identified the valid range for the balancing parameter $\delta$, albeit only for Nievergelt \& Reingold's variant - our proof seems to be the first mechanical verification of Adam's variant. 
Given such thorough analysis of the algorithms, we did not expect to learn anything new about this data structure, and for the most part, this was true. Our proofs are free of any manual calculations about tree sizes and the balancing condition. We just state the proper preconditions for each lemma, and Coq's automation for linear integer arithmetic, lia (Besson, 2006), takes care of the rest.

One exception was the crucial function balancel which is used, according to the documentation "when the left subtree might have been inserted to or when the right subtree might have been deleted from". This suggests the precondition

$$
\left(\operatorname{bal}\left(s_{1}-1, s_{2}\right) \wedge 0<s_{1}\right) \vee \operatorname{bal}\left(s_{1}, s_{2}\right) \vee \operatorname{bal}\left(s_{1}, s_{2}+1\right)
$$

corresponding to the three cases: left tree inserted to, no change, and right tree deleted from. This is also what Hirai and Yamamoto used in their formalization of Nievergelt \& Reingold's variant. And indeed, this precondition is strong enough to verify that the output of balancel is balanced - but we found the precondition is too strong. The link operation, shown in Figure 8, is supposed to balance two arbitrary trees using balancel. In the verification of link we were unable to satisfy this precondition. ${ }^{35}$

We found a precondition for balancel that is both strong enough for the verification of balancel and weak enough to allow the verification of link, namely

$$
\left(\operatorname{bal}^{*}\left(s_{1}-1, s_{2}\right) \wedge 0<s_{1}\right) \vee \operatorname{bal}\left(s_{1}, s_{2}\right) \vee \operatorname{bal}\left(s_{1}, s_{2}+1\right)
$$

where we used the following modified balancing condition for the left tree (the one we inserted into)

$$
\operatorname{bal}^{*}\left(s_{1}, s_{2}\right):=\delta \cdot s_{1} \leq \delta^{2} \cdot s_{2}+\delta \cdot s_{2}+s_{2} \wedge s_{2} \leq s_{1} .
$$

No creativity was involved in coming up with this formula: In the proof of link, in the case where we call balanceL with trees with sizes $s_{1}$ and $s_{2}$, the interactive proof system conveniently gives us all available facts about the sizes of the tress. We put these into a single formula, namely

$\exists s s_{l} s_{r}, s_{1}=1+s+s_{l} \wedge s_{2}=s_{r} \wedge \operatorname{bal}\left(s_{l}, s_{r}\right) \wedge \delta \cdot s<1+s_{l}+s_{r} \wedge 1 \leq s \wedge 0 \leq s_{l} \wedge 0 \leq s_{r}$, and then (manually) eliminated the existential quantifiers to obtain bal* $\left(s_{1}-1, s_{2}\right)$.

\subsection{Verification of big-endian Patricia trees}

There is surprisingly little literature on the verification of big-endian Patricia trees. They are sorted search trees, but as they exploit the additional structure of their keys being bit strings, additional theory is required to verify them.

In particular, where the verification of regular search trees uses open intervals to describe the range of possible values of a tree, Patricia trees build on dyadic intervals. These intervals are of the form $\left[p \cdot 2^{b}, \ldots,(p+1) \cdot 2^{b}-1\right]=: d(p, b)$ for some prefix $p \in \mathbb{N}$ and

35 Indeed, consider a left tree with a size of 5, and a right tree with a size of 17 . And the right tree contains a left subtree of size 12 and a right subtree of size 4 . All the trees are balanced and they satisfy the precondition of link. However, none of the three inequalities in balanceL's precondition can be satisfied with this example. 


$$
\begin{gathered}
r_{1} \subseteq h_{1}(d(p, b)) \quad \begin{array}{cc}
\mathrm{s}_{1}:: r_{1}:: D_{1} & \mathrm{~s}_{2}:: r_{2}:: D_{2} \\
r_{2} \subseteq h_{2}(d(p, b)) & b>0 \quad \mathrm{p}^{\prime}=p \cdot 2^{b} \quad \mathrm{~m}=2^{b-1}
\end{array} \\
{\text { Bin p' } \mathrm{m}_{1} \mathrm{~s}_{2}:: d(p, b):: D_{1} \cup D_{2}}^{\mathrm{p}^{\prime}=p \cdot w} \quad 0<\mathrm{bm}<2^{w} \\
\hline \text { Tip p' bm }:: d\left(p, \log _{2} w\right)::\{p \cdot w+j \mid \text { bit } j \text { set in bm }\}
\end{gathered}
$$

Fig. 10. The denotation of IntSet.

bit-width $b \in \mathbb{N}$, and have an interesting algebraic structure that plays a crucial role in the verification. In particular: ${ }^{36}$

- A dyadic interval is non-empty.

- Either two dyadic intervals are disjoint, or one is contained in the other.

- A dyadic interval $d(p, b)$ with $b>0$ is the disjoint union of its two halves, $h_{1}(d(p, b)):=d(p, b-1)$ and $h_{2}(d(p, b)):=d(p+1, b-1)$.

- In particular, if another dyadic interval $r$ overlaps with with both halves of $d(p, b)$, then $d(p, b) \subseteq r$.

- The dyadic intervals, ordered by inclusion, form a join semi-lattice: for any two dyadic intervals $r_{1}, r_{2}$ there exists a least dyadic interval $r_{1} \sqcup r_{2}$ that contains the two.

The verification of the IntSet library ${ }^{37}$ revolves around the relation $s:: r:: D$, given in Figure 10. This relation expresses that a non-empty tree $s$ of type IntSet describes the set $D$, which is contained in the dyadic interval $r$ :

Lemma 1. If $\mathrm{s}:: r::$, then

- $\emptyset \subset D \subseteq r$ and

- member $\times \mathrm{s}==$ true iff $x \in D$.

We can now specify the operations on Patricia trees in terms of this relation, e.g., the specification for the union operator would be: "if $s_{1}:: r_{1}:: D_{1}$ and $s_{2}:: r_{2}:: D_{2}$, then union $\mathrm{s}_{1} \mathrm{~s}_{2}:: r_{1} \sqcup r_{2}:: D_{1} \cup D_{2}$ ". By including the specification on the associated dyadic interval in the statement of the lemma, a proof by induction has all necessary information about the subterms to conclude.

This theory applies to natural numbers (bounded or unbounded). Negative numbers require additional thought, as the nice algebraic structure of dyadic intervals breaks down: A dyadic interval is either completely negative or completely nonnegative; the two dyadic intervals $r_{1}=d(-1,1)=\{-2,-1\}$ and $r_{2}=d(0,1)=\{0,1\}$ do not have a least upper bound $r_{1} \sqcup r_{2}$. So in order to apply this theory to signed integers, we have to somehow map them to unsigned integers:

36 The Coq formalization of this theory is in the file examples/containers/theories/DyadicIntervals.v

37 In the file examples/containers/theories/IntSetProofs.v. 
- For signed integers of a bounded bit-width $w$ - as encountered in IntSet - the usual encoding of negative numbers two's complement, is suitable. It represents a negative number $i$ by negating all bits of its absolute value $|i|$ and then adding 1 to the result. This effectively maps the negative numbers $\left\{-2^{w-1}, \ldots,-1\right\}$ onto the range $\left\{2^{w-1}, \ldots 2^{w}-1\right\}$. Most operations only use bit-level operations and work just fine. Operations related to order (e.g., toList, findMin) need to treat a Bin constructor that branches on the highest bit specially, as in this case, the right subtree contains the smaller numbers.

- For signed unbounded types, the two's complement representation is unsuitable: It represents -1 as a number with infinitely many bits set, which we cannot just interpret as a unsigned number. In order to build a IntSet-like data structure for signed unbounded types, one would either have to chose a different encoding of natural numbers where all numbers are represented with finitely many bit set (such as signed magnitude representation), or simply use a pair of sets of absolute values, one for the negative and one for the positive numbers.

\subsection{Improvements to containers}

Although our verification did not find bugs in the code of the library, we were able to improve containers: during the verification of Data. Set.union, we noticed that it was using a nested pattern match to check if an argument is a singleton set, when it could be testing if the size was 1 directly. This change turned out to provide a $4 \%$ speedup to union, as measured by the benchmark suite i container, and has been released with containers $0.6 .^{38}$

Additionally, as we mention in Section 4.1, our well-formedness property for IntSet uncovered a weakness in the valid function for IntSet and IntMap, which was used in the test suite. The valid function failed to ensure that some of the invariants hold recursively in the tree structure, which was necessary for the proof. We notified the containers maintainers, ${ }^{39}$ who then made the valid function complete.

We also provide a package, containers-verified, ${ }^{40}$ which re-exports the types and definitions we have verified from the precise version of containers we are working with. This way, a developer who wants to use only the verified portion of the implementation can replace their dependency on containers with a dependency on containers-verified.

\section{Assumptions and limitations}

We have shown a way to make mechanically checked, formal statements about existing Haskell code, and have applied this technique to verify parts of the containers library. But are the theorems that we prove actually true? And if they are, how useful is this method?

38 https://github.com/haskell/containers/commit/b1a05c3a2

39 https://github.com/haskell/containers/issues/522

40 https://hackage.haskell.org/package/containers-verified 


\subsection{The formalization gap}

As always, when a theorem is stated about a object that does not purely exist within mathematics, its validity depends on a number of assumptions.

- First and foremost, we have to assume that Coq behaves as documented and does not allow us to prove false theorems. This is of particular relevance because we rely on fine details of Coq's machinery (e.g., the behavior of Qed, Section 5.4) and optionally add consistent axioms (see Section 5.7.4). We also use the axiom of proof irrelevance, which is consistent with Coq and a consequence of classical logic in the Calculus of Inductive Constructions (Coquand, 1989).

- The biggest assumption is that the semantics of a Gallina program, as defined by the Calculus of Constructions (Coquand \& Huet, 1988), models the behavior of a running Haskell program in a meaningful way. At this time, we cannot even attempt to close this gap, as there are neither formal semantics nor verified compilers for Haskell. While Haskell and Gallina are very similar (both are pure functional languages with algebraic data types), the significant differences between them (such as Haskell's partiality and laziness) mean that the gap is a real presence.

We can gain additional confidence by testing this connection: we extracted our Gallina versions of Set and IntSet back to Haskell and successfully ran the original test suite of containers against it. Every successful test is further evidence that the corresponding theorem (see Section 4.2) is indeed a theorem about the Haskell program.

- Furthermore, we rely on hs-to-coq translating Haskell code into the correct Gallina code. The translator itself is a sizable piece of code, unverified (and such verification is currently out of our reach, not least because there is no formal semantics of Haskell) and therefore surely not free of bugs. We get some confidence into the tool from manually inspecting its output and observing that it is indeed what we would consider the "right" translation from Haskell into Gallina, and additional confidence from the fact that we were actually able to prove the specifications, which would not be possible if the translated code behaved differently than intended. Moreover, extracting the translated code back to Haskell and running the test suite also stress-tests the translation.

- The translation was not completely automatic and required manual edits. With each edit, we add another assumption to the formalization gap: Does our underspecification of pointer equality encompass the actual behavior of GHC's reallyUnsafePtrEquali ty\#? Given our choice of using unbounded integers, does the size field in a Set really never overflow in practice? Are our manually written versions of low-level bit-twiddling functions correct? We list and justify our manual interventions in Section 5.

The formalization gap of our work is relatively large compared to, say, the gap for the verification of programs written in Gallina in the first place. But for the purpose of ensuring the correctness of the Haskell code, that is less critical. Even if one of our assumptions is flawed, it is much more likely that the flaw will get in the way of concluding the proofs, rather than allowing us to conclude the proofs without noticing a bug. Incomplete proofs can uncover bugs, too. 


\subsection{Limitations of our approach}

We proved multiple specifications about a large part of the code, but there are limits to what theorems we can prove, and what we can prove them about.

Since we work with a shallow embedding of Haskell in Coq, we cannot make statements about the performance of the Haskell code - which is a pity, given that the containers library contains highly optimized code and provides clear specifications of the algorithmic complexity of their operations. Similarly, we cannot verify that the operations are as strict or lazy as documented.

We also cannot translate and verify all code in the containers library, because some functions use language features not yet supported by hs-to-coq, such as mutual recursion and unboxed arrays. When this affects a crucial utility function we can provide a manual translation. This widens the formalization gap, but enables verification of code that depends on it. When it affects less central code, e.g., the Data instances, we can simply skip the translation (Section 5.3).

Partiality is a particularly interesting limit. Coq only allows total functions, but practical Haskell code uses partiality, often in a benign way: Calls to error in code paths that are unreachable as long as invariants are maintained, or recursive functions that terminate on the actual arguments they are called with, but may diverge on other inputs. Whereas Spector-Zabusky et al. (2018) considered such code out of scope, we have found ways to deal with "internal partiality" (see Section 5.4 and 5.7.4).

Taking a step back, it might seem that our approach may see limited adoption in the Haskell community because it requires expertise in Coq. But though tied to a Haskell artifact, verification is isolated from the codebase. Haskell programmers can focus on their domain, trying to get the best performance out of the code and without having to know about verification, while proof engineers can work solely within Coq and do not have to be fluent in Haskell.

In this paper we verify a specific version of the Haskell code, and do not discuss ongoing maintenance of such a verification. It remains to be seen how resilient the proofs are against changes in the Haskell code. Changes of syntactic nature, or changes to a function's strictness, might be swallowed by hs-to-coq. Other changes might affect the translated code, but still allow the proofs to go through, if our proof tactics are flexible enough. In general, though, we expect that changes in the code require changes in the proofs. Since Coq is an interactive theorem prover, it will at least clearly point out which parts of our development need to be updated.

\section{Related work}

\subsection{Verification of purely functional data structures}

Purely functional data structures, such as those found in Okasaki's book (1999) are frequent targets of mechanical verification. That said, we believe that we are the first to verify the Patricia tree algorithms that underlie Data. IntSet. 
Verifying weight-balanced trees in Haskell. Similar to hs-to-coq, LiquidHaskell (Vazou et al., 2014) can be used to verify existing Haskell code. Users of this tool annotate their Haskell source files with refinement types and other annotations. LiquidHaskell then uses an SMT solver to automatically discharge proof obligations described by the refinements. This means that LiquidHaskell provides more automation than hs-to-coq; however, the language of Coq is higher order and more expressive than the language of SMT solvers. Vazou et al. (2017) compared the experiences of using LiquidHaskell as apposed to plain Coq, and found that both have advantages.

Vazou et al. (2013) also described the use of LiquidHaskell to verify the Data. Map module of finite maps from containers. Although not the same as Data. Set, this code shares the same underlying data structure (weight-balanced trees) and the implementations of the two are similar. Their verification has similarities with our work; they also use unbounded integers as the number representation and leave functions like showTree unspecified. However, we develop a richer specification, which includes a semantic description of each operation we verified, constraints about the tree balance, and the ordering of the elements in the tree. In contrast, Vazou et al. limit their specifications to ordering only. For example, in addition to showing that the insertion operation preserves the order of elements of the tree, our work also shows that: (1) insertion preserves the balancing conditions of the weight-balanced tree, (2) the size field at each node in the tree is maintained correctly (i.e., the size is equal to the number of its descendants), and (3) the tree returned by this operation contains the inserted element, and all elements in the original tree, but nothing more. Although it might be possible to replicate our specifications by using theories of finite sets and maps in SMT (Kröning et al., 2009) to encode these properties using refinement types in LiquidHaskell, this approach has not been explored.

Furthermore, our specification also includes type class laws, and we are able to verify that Set and IntSet have lawful instances of the Eq, Ord, Semigroup, and Monoid type classes. When Vazou et al.'s original work was developed in 2013, LiquidHaskell did not have the capability to state and prove these properties. Since then, there have been new developments in LiquidHaskell, particularly refinement reflection (Vazou et al., 2018), which could make it possible to specify and prove type class laws.

Both LiquidHaskell and hs-to-coq check for termination of Haskell functions. In LiquidHaskell, the termination check is an option that can be deactivated, allowing the sound verification of nonstrict, non-terminating functions (Vazou et al., 2014). In contrast, a proof of termination is a requirement for verifying functions using hs-to-coq (SpectorZabusky et al., 2018). However, hs-to-coq is able to take advantage of many options available in Coq for proving termination of nontrivial recursion, including structural recursion, Program Fixpoint and our own approach based on deferredFix. This latter approach allowed us to reason about fromDistinctAscList (see Section 5.7.4) and prove that it is indeed terminating; on the other hand, Vazou et al. deactivate the termination check for this function.

Verifying weight-balanced trees in other languages. Hirai \& Yamamoto (2011) implemented a weight-balanced tree similar to Haskell's Data. Set library (albeit using the balancing condition of Nievergelt \& Reingold (1972)) and mechanically verified its balancing properties in Coq. More recently, Nipkow \& Dirix (2018) extended this work 
to formalize similar weight-balanced trees in Isabelle and further verified the functional correctness of insertions and deletions.

We verify more functions in the Data. Set library than prior work. Operations that are unique to our development include foldl, isSubsetOf, and fromDistinctAscList. The code verified both by Hirai \& Yamamoto and by Nipkow \& Dirix is also different from the latest containers library; for example, it does not use pointer equality (Section 5.1). Another difference is that Hirai \& Yamamoto defined the union, intersection, and difference functions based on the "hedge union" algorithm, but containers has since changed to use the "divide and conquer" algorithm.

Hirai \& Yamamoto specify only the balancing constraints, whereas we develop a richer specification that also includes a semantic description of each operation we verified and the ordering of the elements in the tree. We also gained new insights about the balancing conditions of the weight-balanced tree through our verification effort (see Section 6.1). Nipkow \& Dirix's specification contains the same properties as ours, but they only specify the behavior of insert and delete; we have verified a significantly larger set of functions (see Figure 4).

Other verifications of balanced trees. There are many existing works on mechanically verifying purely functional balanced trees. We briefly mention a few here. Filliâtre \& Letouzey (2004) implemented AVL trees in Coq, and verified their functional correctness as well as their balancing conditions. Appel (2011) did the same thing for red-black trees. These implementations have now become parts of Coq standard library. Charguéraud (2010b) translated OCaml implementations of Okasaki (1999)'s functional data structures to characteristic formulae expressed as Coq axioms. Licata (2012) lectured on verifying red-black trees in Agda at the Oregon Programming Languages Summer School. McBride (2014) showed how to represent the ordering relationships in Agda for general data structures, not just binary search trees. Nipkow (2016) showed how to automatically verify the ordering properties of eight different binary search tree structures, by specifying each in terms of the sorted list of their elements, a method he used again in his verification of weight-balanced trees (Nipkow \& Dirix, 2018). Ralston (2009) verified AVL trees in ACL2.

\subsection{Verification tools for Haskell}

Previous work using hs-to-coq has only applied it to small examples. Spector-Zabusky et al. (2018) describe three case studies, two of which require less than 20 lines of Haskell. The longest example (the Bag module taken directly from the GHC compiler) is 247 lines of code. Furthermore, none of the reasoning required for these examples is particularly deep. Our work provides experience with more complex, externally sourced, industrialstrength examples.

The coq-haskell library (Wiegley, 2017) is a handwritten Coq library designed to make it easier for Haskell programmers to work in Coq. In addition to enabling easier Coq programming, it also provides support for extracting Coq programs to Haskell.

The prototype contract checker halo (Vytiniotis et al., 2013) takes a Haskell program, uses GHC to desugar it into the intermediate language Core, and translates the Core 
program into a first-order logic formula. It then invokes an SMT solver to prove this formula; a successful proof implies that the original program is crash-free.

Haskabelle was hs-to-coq's counterpart in Isabelle. It translated total Haskell code into equivalent Isabelle function definitions. Similar to hs-to-coq, it parsed Haskell, desugared syntactic constructs, and configurably adapted basic types and functions to their counterparts in Isabelle's standard library. It used to be bundled with the Isabelle release, but it has not been updated recently and was dropped from the distribution.

Haskell has been used as a prototyping language for mechanically verified systems in the past. The seL4 verified microkernel started with a Haskell prototype that was semiautomatically translated to Isabelle/HOL (Klein et al., 2009; Derrin et al., 2006). The authors found that the availability of the Haskell prototype provided a machine-checkable formal executable specification of the system. They used this prototype to refine their designs via testing, allowing them to make corrections before full verification.

The Programmatica project (Hallgren et al., 2004) included a tool that translates Haskell code into the Alfa proof editor. Their tool only produces valid proofs for total functions over finite data structures. The logic of the Alfa proof assistant is based on dependent type theory, but without as many features as Coq. In particular, the Programmatica tool compiles away type classes and nested pattern matching; both of these features are retained by hs-to-coq.

Dybjer et al. developed a tool for automatically translating Haskell programs to the Agda/Alfa proof assistant (2004). They explicitly note the interplay between testing and theorem proving and show how to verify a tautology checker. Abel et al. (2005) translate Haskell expressions into the logic of the Agda 2 proof assistant. Their tool works later in the GHC pipeline than hs-to-coq and translates Core expressions.

\subsection{Translating other higher order functional languages}

There are many large and successful verification projects that demonstrate that functional languages are well suited for verification. In contrast to our work, these projects require either re-implementing the code in a new functional language, as is the case for Cogent (O'Connor et al., 2016; Amani et al., 2016) and F* (Swamy et al., 2016); re-implementing the code in a proof assistant, such as HOL4 in the case of CakeML (Myreen \& Owens, 2014; Kumar et al., 2014); or taking an SMT solver-based approach, as found in Leon (Blanc et al., 2013). The CakeML and Cogent projects have a different focus than ours, and they provide a higher assurance in their verified code. CakeML (Kumar et al., 2014) has a verified compiler and Cogent has a certifying compiler (O'Connor et al., 2016; Rizkallah et al., 2016). Both tools provide mechanically checked proofs that their shallow embeddings correspond to the functional code being verified.

Cogent is a restricted higher order functional language (O'Connor et al., 2016) that was used to verify filesystems (Amani et al., 2016). Its compiler produces $\mathrm{C}$ code, a highlevel specification in Isabelle/HOL, and an Isabelle/HOL refinement proof linking the two (O’Connor et al., 2016; Rizkallah et al., 2016). Chen et al. (2017) integrated propertybased testing into the Cogent framework. The authors claim that property-based testing enables an incremental approach to a fully verified system, as it allows for the replacement of tests of properties stated in the specification by formal proofs. Our work substantiates 
this claim, as indeed one of the ways in which we obtain specifications is through the QuickCheck properties provided by Haskell as discussed in Section 4.2.

CakeML is a large subset of ML with a verified compiler and runtime system (Kumar et al., 2014). Users of CakeML can write pure functional programs in the HOL4 proof assistant and verify them in HOL4. They can then extract an equivalent correct by construction CakeML program. This method has been used to verify several data structures including red black trees, crypto protocols, and a CakeML version of the HOL light theorem prover (Myreen \& Owens, 2014).

$F^{*}$ is a general-purpose functional language that allows for a mixture of proving and general-purpose programming (Swamy et al., 2016). Programs can be specified using dependent and refinement types and automatically verified using one of $\mathrm{F}^{*}$ 's backend SMT solvers. Its subset Low* (Protzenko et al., 2017) has been used to verify high-assurance optimized cryptographic libraries.

Leon is a verification tool for a pure subset of Scala that checks pre- and postconditions for functions (Blanc et al., 2013). These contracts are given in terms of the requires and ensures methods that are already present in the Scala standard library, so a sufficiently well-annotated existing Scala program would need no further specification. Leon uses Z3 to ensure that the contracts are satisfied, and is complete - if a counterexample exists, it will be found. It also supports extensions, allowing Leon to verify a larger subset of Scala through translation into a simpler form.

\subsection{Extraction}

The semantic proximity of Haskell and Coq, which we rely on, is also used in the other direction by Coq's support for code extraction to Haskell (Letouzey, 2002). Several projects use this feature to verify Haskell code (Chen et al., 2015; Joseph, 2014). However, since extraction starts with Coq code and generates Haskell, it cannot be used to verify preexisting Haskell. Although in a certain sense hs-to-coq and extraction are inverses, round-tripping does not produce syntactically equivalent output in either direction. In one direction, hs-to-coq extensively annotates the resulting Coq code; in the other, extraction ignores many Haskell features and inserts unsafe type coercions. In this work, we use testing to verify that round-tripping produces operationally equivalent output; this provides assurance about the correctness of both hs-to-coq and extraction.

CertiCoq (Anand et al., 2017) and Euf (Mullen et al., 2018) are verified compilers from Gallina to assembly. CertiCoq can compile any Gallina program, while Euf can only compile a limited subset of Gallina. However, Euf provides stronger guarantees about the Gallina code that is in the limited subset it translates.

\section{Conclusions and future work}

We verified the two finite set modules that are part of the widely used and highly optimized containers library. Our efforts provide the deepest specification and verification of this code to date, covering more of the API and proving stronger, more descriptive properties than prior work. 
In future work, there is yet more to verify in containers. For example, we plan to add a version of IntSet that uses 64-bit ints as the element type in addition to our current version with unbounded natural numbers. That way users could choose the treatment of overflow that makes the most sense for their application. Furthermore, we hope to adapt our existing proofs of IntSet to the analogous Data. IntMap module.

The fact that we did not find bugs says a lot about the tools that are already available to Haskell programmers for producing correct code, such as a strong, expressive type system and a mature property-based testing infrastructure. However, few would dare to extrapolate from these results to say that all Haskell programs are bug free! Instead, we view verification as a valuable opportunity for functional programmers and an activity that we hope will become more commonplace.

\section{Acknowledgments}

We thank the anonymous JFP reviewers whose suggestions helped clarify and improve this paper. This material is based upon work supported by the National Science Foundation under Grant No. 1319880 and Grant No. 1521539.

\section{Conflicts of interest}

None.

\section{References}

Abel, A., Benke, M., Bove, A., Hughes, J., \& Norell, U. (2005) Verifying Haskell programs using constructive type theory. In Haskell Workshop. ACM, pp. 62-73.

Adams, S. (1992) Implementing sets efficiently in a functional language,. Research Report CSTR 92-10. University of Southampton.

Amani, S., Hixon, A., Chen, Z., Rizkallah, C., Chubb, P., O’Connor, L., Beeren, J., Nagashima, Y., Lim, J., Sewell, T., Tuong, J., Keller, G., Murray, T., Klein, G. \& Heiser, G. (2016) Cogent: Verifying high-assurance file system implementations. In International Conference on Architectural Support for Programming Languages and Operating Systems, pp. 175-188.

Anand, A., Appel, A., Morrisett, G., Paraskevopoulou, Z., Pollack, R., Savary Belanger, O., Sozeau, M. \& Weaver, M. (2017) CertiCoq: A verified compiler for Coq. In CoqPL Workshop, CoqPL 2017.

Appel, A. W. (2011) Efficient Verified Red-Black Trees.

Appel, A. W., Beringer, L., Chlipala, A., Pierce, B. C., Shao, Z., Weirich, S. \& Zdancewic, S. (2017) Position paper: The science of deep specification. Philos. Trans. R. Soc. A 375(2104).

Besson, F. (2006) Fast reflexive arithmetic tactics: the linear case and beyond. In TYPES. Lecture Notes in Computer Science, vol. 4502. Springer, pp. 48-62.

Blanc, R., Kuncak, V., Kneuss, E. \& Suter, P. (2013) An overview of the Leon verification system: verification by translation to recursive functions. In Proceedings of the 4th Workshop on Scala, SCALA@ECOOP 2013, Montpellier, France, July 2, 2013. ACM, pp. 1:1-1:10.

Bove, A., Dybjer, P. \& Norell, U. (2009) A brief overview of Agda - A functional language with dependent types. In: Theorem Proving in Higher Order Logics, 22nd International Conference, TPHOLs 2009, Munich, Germany, August 17-20, 2009. Proceedings, Berghofer, S., Nipkow, T., Urban, C. \& Wenzel, M. (eds), Lecture Notes in Computer Science, vol. 5674. Springer. 
Brady, E. (2017) Type-driven development with Idris. Manning.

Campbell, T. (2010) Bug in Data.Map. e-mail to the Haskell libraries mailing list.

Charguéraud, A. (2010a) The optimal fixed point combinator. In Proceedings of the First International Conference on Interactive Theorem Proving. ITP 2010. Berlin, Heidelberg: Springer-Verlag, pp. 195-210.

Charguéraud, A. (2010b) Program verification through characteristic formulae. In ICFP. ACM, pp. 321-332.

Chen, H., Ziegler, D., Chajed, T., Chlipala, A., Kaashoek, M. F. \& Zeldovich, N. (2015) Using Crash Hoare logic for certifying the FSCQ file system. SOSP. ACM, pp. 18-37.

Chen, Z., O'Connor, L., Keller, G., Klein, G. \& Heiser, G. (2017) The Cogent case for propertybased testing. Workshop on Programming Languages and Operating Systems (PLOS). Shanghai, China: ACM, pp. 1-7.

Claessen, K. \& Hughes, J. (2000) QuickCheck: A lightweight tool for random testing of Haskell programs. ICFP, ACM, pp. 268-279.

Coquand, T. (1989) Metamathematical investigations of a calculus of constructions. Tech. rept. RR1088. INRIA.

Coquand, T. \& Huet, G. P. (1988) The calculus of constructions. Information and computation, 76(2/3), 95-120.

Derrin, P., Elphinstone, K., Klein, G., Cock, D. \& Chakravarty, M. M. T. (2006) Running the manual: An approach to high-assurance microkernel development. In Haskell Symposium. ACM, pp. 60-71.

Dybjer, P., Haiyan, Q. \& Takeyama, M. (2004) Verifying Haskell programs by combining testing, model checking and interactive theorem proving. Inform. Softw. Technol. 46(15), 1011-1025.

Filliâtre, J.-C. \& Letouzey, P. (2004) Functors for proofs and programs. In Programming Languages and Systems, Schmidt, D. (ed). Berlin, Heidelberg: Springer Berlin Heidelberg, pp. 370-384.

Hallgren, T., Hook, J., Jones, M. P. \& Kieburtz, R. B. (2004) An overview of the Programatica toolset. In HCSS.

Hirai, Y. \& Yamamoto, K. (2011) Balancing weight-balanced trees. J. Function. Program. 21(3), 287-307.

Joseph, A. M. (2014) Generalized arrows. Ph.D. thesis, EECS Department, University of California, Berkeley.

Klein, G., Elphinstone, K., Heiser, G., Andronick, J., Cock, D., Derrin, P., Elkaduwe, D., Engelhardt, K., Kolanski, R., Norrish, M., Sewell, T., Tuch, H. \& Winwood, S. (2009) seL4: Formal verification of an OS kernel. In ACM Symposium on Operating Systems Principles. Big Sky, MT, USA: ACM, pp. 207-220.

Krauss, A. (2006) Partial recursive functions in higher-order logic. In IJCAR. LNCS, vol. 4130. Springer, pp. 589-603.

Kröning, D., Rümmer, P. \& Weissenbacher, G. (2009) A proposal for a theory of finite sets, lists, and maps for the SMT-Lib standard. In Informal Proceedings, 7th International Workshop on Satisfiability Modulo Theories at CADE, vol. 22.

Kumar, R., Myreen, M. O., Norrish, M. \& Owens, S. (2014). CakeML: A verified implementation of ML. In Proceedings of the 41st ACM SIGPLAN-SIGACT Symposium on Principles of Programming Languages, POPL 2014. New York, NY, USA: ACM, pp. 179-191.

Letouzey, P. (2002) A new extraction for Coq. In TYPES. LNCS, vol. 2646. Springer, pp. 200-219.

Licata, D. (2012) 15-150 Lecture 21: Red-black trees. Lecture at the Oregon Programming Language Summer School.

The Coq development team. (2016) The Coq proof assistant reference manual. LogiCal Project. Version 8.6.1.

McBride, C. T. (2014) How to keep your neighbours in order. In Proceedings of the 19th ACM SIGPLAN International Conference on Functional Programming, ICFP 2014. New York, NY, USA: ACM, pp. 297-309.

Morrison, D. R. (1968) PATRICIA-Practical Algorithm To Retrieve Information Coded in Alphanumeric. J. ACM 15(4), 514-534. 
Mullen, E., Pernsteiner, S., Wilcox, J. R., Tatlock, Z. \& Grossman, D. (2018) Euf: Minimizing the Coq extraction TCB. In Proceedings of the 7th ACM SIGPLAN International Conference on Certified Programs and Proofs, CPP 2018. New York, NY, USA: ACM, pp. 172-185.

Myreen, M. O. \& Owens, S. (2014) Proof-producing translation of higher-order logic into pure and stateful ML. Journal of Functional Programming, 24(May), 284-315.

Nievergelt, J. \& Reingold, E. M. (1972) Binary search trees of bounded balance. In STOC. ACM, pp. 137-142.

Nipkow, T. (2016) Automatic functional correctness proofs for functional search trees. In Interactive Theorem Proving (ITP) 2016, Blanchette, J. \& Merz, S. (eds), vol. 9807, pp. 307-322.

Nipkow, T. \& Dirix, S. (2018) Weight-balanced trees. In Archive of Formal Proofs, http:// isa-afp.org/entries/Weight_Balanced_Trees.html, Formal proof development.

Nipkow, T., Paulson, L. C. \& Wenzel, M. (2002) Isabelle/HOL - A Proof Assistant for Higher-Order Logic. Lecture Notes in Computer Science, vol. 2283. Springer.

O’Connor, L., Chen, Z., Rizkallah, C., Amani, S., Lim, J., Murray, T., Nagashima, Y., Sewell, T. \& Klein, G. (2016) Refinement through restraint: Bringing down the cost of verification. International Conference on Functional Programming.

Okasaki, C. (1999) Purely Functional Data Structures. Cambridge University Press.

Okasaki, C. \& Gill, A. (1998) Fast mergeable integer maps. In Workshop on ML, pp. 77-86.

Peyton Jones, S., Tolmach, A. \& Hoare, T. (2001) Playing by the rules: rewriting as a practical optimisation technique in GHC. In Haskell Workshop.

Protzenko, J., Zinzindohoué, J.-K., Rastogi, A., Ramananandro, T., Wang, P., Zanella-Béguelin, S., Delignat-Lavaud, A., Hriţcu, C., Bhargavan, K., Fournet, C. \& Swamy, N. (2017) Verified low-level programming embedded in $\mathrm{F}^{*}$. Proc. ACM program. lang., 1(ICFP), 17:1-17:29.

Ralston, R. (2009) ACL2-certified AVL trees. In Proceedings of the Eighth International Workshop on the ACL2 Theorem Prover and Its Applications, ACL2 2009. New York, NY, USA: ACM, pp. 71-74.

Rizkallah, C., Lim, J., Nagashima, Y., Sewell, T., Chen, Z., O’Connor, L., Murray, T., Keller, G. \& Klein, G. (2016) A framework for the automatic formal verification of refinement from Cogent to C. In International Conference on Interactive Theorem Proving.

Spector-Zabusky, A., Breitner, J., Rizkallah, C. \& Weirich, S. (2018) Total Haskell is reasonable Coq. In CPP. ACM, pp. 14-27.

Straka, M. (2010) The performance of the Haskell CONTAINERS package. Proceedings of the Third ACM Haskell Symposium on Haskell, Haskell 2010. New York, NY, USA: ACM, pp. 13-24.

Swamy, N., Hriţcu, C., Keller, C., Rastogi, A., Delignat-Lavaud, A., Forest, S., Bhargavan, K., Fournet, C., Strub, P.-Y., Kohlweiss, M., Zinzindohoue, J.-K. \& Zanella-Béguelin, S. (2016) Dependent types and multi-monadic effects in $\mathrm{F}^{*}$. Proceedings of the 43rd Annual ACM SIGPLAN-SIGACT Symposium on Principles of Programming Languages, POPL 2016. New York, NY, USA: ACM, pp. 256-270.

Vazou, N., Rondon, P. M. \& Jhala, R. (2013) Abstract refinement types. In Proceedings of the 22nd European Conference on Programming Languages and Systems, ESOP 2013. Berlin, Heidelberg: Springer-Verlag, pp. 209-228.

Vazou, N., Seidel, E. L., Jhala, R., Vytiniotis, D. \& Peyton-Jones, S. (2014) Refinement types for Haskell. ICFP. ACM, pp. 269-282.

Vazou, N., Lampropoulos, L. \& Polakow, J. (2017) A tale of two provers: Verifying monoidal string matching in Liquid Haskell and Coq. In Haskell Symposium. ACM, pp. 63-74.

Vazou, N., Tondwalkar, A., Choudhury, V., Scott, R. G., Newton, R. R., Wadler, P. \& Jhala, R. (2018) Refinement reflection: Complete verification with SMT. PACMPL, 2(POPL), 53:1-53:31.

Vytiniotis, D., Peyton Jones, S., Claessen, K. \& Rosén, D. (2013) HALO: Haskell to logic through denotational semantics. In POPL. ACM, pp. 431-442.

Wiegley, J. (2017) coq-haske l l: A Library for Formalizing Haskell Types and Functions in Coq. https://github.com/jwiegley/coq-haskell. 